\title{
Álgebras com Composição e Álgebras com Pseudo-Composição
}

Walquiria de Freitas Torezani

DISSERTAÇÃO APRESENTADA

AO

INSTITUTO DE MATEMÁTICA E ESTATÍSTICA

DA

UNIVERSIDADE DE SÃO PAULO

PARA CBTENÇÃO DO GRAU

DE

MESTRE EM MATETAATICA

Área de Concentração: Ålgebra

Oricatador: Prof. Dr. Luiz Antonio Peresi

Durante a elaboração deste trabalho,

a autora recebe apoio financeiro da CAPES.

São Paulo, junho de 1998 


\section{Álgebras com Composição \\ e \\ Álgebras com Pseudo-Composição}

Este exemplar corresponde à redação final da dissertação apresentada por Walquiria de Freitas Torezani, aprovada pela Banca Examinadora.

Banca Examinadora:

Prof. Dr. Luiz Antonio Peresi (orientador) IME-USP

Prof. Dr. Orlando Stanley Juriaans IME-USP

Prof. Dr. Antonio Paques IMECC-UNICAMP 
A Darci, Marcus e Marcelo. 


\section{Agradecimentos}

Acima de todos, a Deus que nunca me desamparou.

A minha família, pelo apoio constante.

Ao meu orientador, o Professor Dr. Luiz Antonio Peresi, por toda a atenção, dedicação e pelo incentivo desde o início do curso de mestrado.

A todos os meus colegas do IME que muito me ajudaram nas dificuldades e alegraramme nos momentos tristes.

Agradeço especialmente ao Daniel Vendrúsculo pelos conselhos e pela força, ao Fernando Martins Antoneli Júnior por todo o carinho e tranqüilidade que me transmitiu, ao Samuel Gomes da Silva por me fazer sorrir nas horas em que um sorriso era algo quase impossível, ao Sidnei Azevedo de Souza por toda a paciência que teve comigo e as colegas Adriana Pádua Lovatte e Elvira Pádua Lovatte pela ajuda na digitação e correção deste trabalho.

Agradeço também àqueles que direta ou indiretamente, desde os tempos da graduação, contribuiram para a realização deste trabalho.

Finalmente, agradeço ao Programa PET do qual fui bolsista desde a graduação e ao Departamento de Matemática da Universidade Federal do Espírito Santo pelo apoio técnico durante a elaboração deste trabalho. 


\section{sumário}

1 Álgebras com Composição 1

1.1 Introdução . . . . . . . . . . . . . . . . . . . 1

1.2 Definição e propriedades simples das álgebras com composição . . . . . 3

$1.3 \mathrm{O}$ processo de Cayley-Dickson . . . . . . . . . . . . . 11

1.4 Exemplos de álgebras com composição . . . . . . . . . . . . . . . 16

1.4.1 Tipo I - Corpos de característica diferente de $2 . \ldots . . . . .16$

1.4.2 Tipo II - Álgebra dos números complexos generalizados. . . . . 17

1.4.3 Tipo III - Álgebra dos quatérnios generalizados. . . . . . . . . 20

1.4.4 Tipo IV - Álgebra de Cayley-Dickson. . . . . . . . . . . . . . . . 20

1.5 Classificação das álgebras com composição . . . . . . . . . . . . . 20

2 Álgebras com Pseudo-Composição $\quad 35$

2.1 Introdução . . . . . . . . . . . . . . . . 35

2.2 Decomposição de Peirce . . . . . . . . . . . . . . . . . 36

2.3 o caso quadrático . . . . . . . . . . . . . 55

2.4 Decomposição de Peirce no caso não quadrático . . . . . . . . . . . 62 


\section{Introdução}

O presente trabalho tem por finalidade classificar as álgebras com composição e as álgebras com pseudo-composição.

No primeiro capítulo, descrevemos o processo de Cayley-Dickson para extensão de uma álgebra e partindo de um corpo $F$ de característica diferente de 2 e de uma forma quadrática estritamente não-degenerada, construimos as álgebras dos complexos generalizados, dos quatérnios generalizados e de Cayley-Dickson que têm dimensão 2, 4 e 8 respectivamente. Provamos que qualquer álgebra com composição é isomorfa ao corpo $F$ ou a uma das álgebras citadas acima. Provamos também que as únicas álgebras com composição que são associativas e comutativas são os corpos de característica diferente de 2 e as álgebras dos complexos generalizados, que as álgebras dos quatérnios generalizados são comutativas mas não são associativas e que as álgebras de CayleyDickson não são nem comutativas nem associativas.

No segundo capítulo estudamos as álgebras com pseudo-composição sobre um corpo algebricamente fechado de característica diferente de 2 e 3 . Tais álgebras necessariamente têm um idempotente $e$. O objetivo principal é a classificação das álgebras com pseudo-composição que não são do tipo quadrático. Mostraremos que essas álgebras podem ser construídas a partir das álgebras alternativas quadráticas com composição. Especificamente mostraremos o seguinte teorema:

Seja A uma álgebra com pseudo-composição com idempotente e sobre um corpo algebricamente fechado $F$ de característica diferente de 2 e 3. Então $\operatorname{Rad} \varphi$ é um ideal nilpotente de A, e um dos seguintes casos acontece:

(a) A é do tipo quadrático,

(b) $A / \operatorname{Rad} \varphi$ é do tipo quadrático,

(c) A pode ser construída a partir de uma álgebra alternativa quadrática com composição. 


\section{Capítulo 1}

\section{Álgebras com Composição}

\subsection{Introdução}

Uma das mais importantes propriedades dos números complexos é expressada pela identidade

$$
\left|z z^{\prime}\right|=|z|\left|z^{\prime}\right|
$$

Se $z=a_{1}+a_{2} i, z^{\prime}=b_{1}+b_{2} i$, então essa identidade é reescrita na forma

$$
\left(a_{1}^{2}+a_{2}^{2}\right)\left(b_{1}^{2}+b_{2}^{2}\right)=\left(a_{1} b_{1}-a_{2} b_{2}\right)^{2}+\left(a_{1} b_{2}+a_{2} b_{1}\right)^{2} .
$$

Essa fórmula pode ser interpretada como: o produto da soma de dois quadrados pela soma de dois quadrados é ainda a soma de dois quadrados.

A pergunta que naturalmente surge é: existem identidades análogas para números maiores de quadrados? Como são descritas tais identidades?

Exemplos de identidades para a soma de quatro quadrados foram exibidos por Euler e Lagrange. Em 1818 Degen encontrou o primeiro exemplo de uma identidade para a soma de oito quadrados que, naquela época, infelizmente, passou despercebido. Em 1843 Hamilton notou que a existência de uma identidade para a soma de $n$ quadrados é 


\subsection{Definição e propriedades simples das álgebras com composição}

Seja $F$ um corpo arbitrário e $A$ um espaço vetorial de dimensão diferente de zero sobre $F$. Uma aplicação $f: A \times A \rightarrow F$ é chamada uma forma bilinear se para todo $x, x^{\prime}, y, y^{\prime} \in A$ e $\alpha \in F$ valem:

(i) $f\left(x+x^{\prime}, y\right)=f(x, y)+f\left(x^{\prime}, y\right)$,

(ii) $f\left(x, y+y^{\prime}\right)=f(x, y)+f\left(x, y^{\prime}\right)$,

(iii) $f(\alpha x, y)=\alpha f(x, y)$.

Dizemos que uma forma bilinear $f$ é simétrica se $f(x, y)=f(y, x)$ para todo $x, y \in A$. Dizemos que $f$ é não-degenerada se:

$$
f(a, x)=0, \quad \forall x \in A \Rightarrow a=0 .
$$

Uma aplicação $n: A \rightarrow F$ é dita uma forma quadrática se:

(i) $n(\lambda x)=\lambda^{2} n(x)$ onde $x \in A, \lambda \in F$,

(ii) A função $f(x, y)=n(x+y)-n(x)-n(y)$ é uma forma bilinear em $A$.

Dizemos que uma forma quadrática $n(x)$ é estritamente não-degenerada se a forma bilinear simétrica $f(x, y)$ associada a $n(x)$ é não degenerada, e dizemos que $n(x)$ é não-degenerada se:

$$
n(a)=0 \varkappa f(a, x)=0, \quad \forall x \in A \Rightarrow a=0 .
$$

Lema 1.1.Toda forma quadrática estritamente não-degenerada é não-degenerada e a recíproca é verdadeira se car $F \neq 2$.

Prova. Seja $n(x)$ uma forma estritamente não-degenerada. Por definição a forma bilinear associada $f(x, y)$ é não-degenerada. Logo se $f(a, x)=0$ para todo $x \in A$ então 
com composiçẫo se:

(i) $n(x y)=n(x) n(y)$, para todo $x, y \in A$,

(ii) a forma $n(x)$ é estritamente não-degenerada,

(iii) existe um elemento identidade $1 \mathrm{em} A$.

Proposição 1.1. Seja $n(x)$ uma forma quadrática estritamente não-degenerada em um espaço vetorial de dimensão finita $A$. Então se $n(x)$ admite algum tipo de composição em $A, n(x)$ admite uma composição x.y com respeito a qual $A$ é uma álgebra com composição.

Prova. Por hipótese a álgebra $A$ com a multiplicação $x y$ e a forma $n(x)$ satisfaz as condições $(i)$ e $(i i)$. Por $(i i)$ existe $a \in A \operatorname{com} n(a) \neq 0$. Seja $u=\frac{a^{2}}{n(a)}$, então

$$
n(u)=n\left(\frac{a^{2}}{n(a)}\right)=\frac{1}{n^{2}(a)} n\left(a^{2}\right)=\frac{n^{2}(a)}{n^{2}(a)}=1 .
$$

Conseqüentemente $n(x u)=n(u x)=n(x)$ e daí,

$$
f(x u, y u)=f(u x, u y)=f(x, y) .
$$

Consideremos agora as aplicações lineares $R_{u}: x \mapsto x u$ e $L_{u}: x \mapsto u x$. Se $R_{u}(x)=0$, então $x u=0$ e daí segue que $f(x, y)=f(x u, y u)=0$, donde concluímos que $x=0$. Analogamente se $L_{u}(x)=0$, então $x=0$. Portanto $R_{u}$ e $L_{u}$ são não-singulares e como $\operatorname{dim} A<\infty$ essas aplicações são invertíveis.

Vamos definir agora uma nova multiplicação em $A$ dada por:

$$
x . y=R_{u}^{-1}(x) L_{u}^{-1}(y) .
$$

Temos que

$$
u^{2} . x=R_{u}^{-1}\left(u^{2}\right) L_{u}^{-1}(x)=u L_{u}^{-1}(x)=x
$$

$\mathrm{e}$

$$
x . u^{2}=R_{u}^{-1}(x) L_{u}^{-1}\left(u^{2}\right)=R_{u}^{-1}(x) u=x .
$$


Isto é,

$$
n(x) f(y, w)=f(x y, x w) .
$$

Analogamente, substituindo $x$ por $x+z$ em (1.1) obtemos

$$
n(y) f(x, z)=f(x y, z y) .
$$

Daí segue que

$$
\begin{aligned}
f(x, z) f(y, w) & =f(x, z)(n(y+w)-n(y)-n(w)) \\
& =f(x, z) n(y+w)-f(x, z) n(y)-f(x, z) n(w) \\
& =f(x(y+w), z(y+w))-f(x y, z y)-f(x w, z w) \\
& =f(x y, z w)+f(x w, z y) .
\end{aligned}
$$

Isto é,

$$
f(x, z) f(y, w)=f(x y, z w)+f(x w, z y) .
$$

Fazendo $z=1$ e $y=x u$ em (1.3) temos

$$
f(x, 1) f(x u, w)=f(x(x u), w)+f(x w, x u) .
$$

Substituindo (1.2) em (1.4) obtemos

$$
f(x(x u), w)+n(x) f(w, u)-f(x, 1) f(x u, w)=0
$$

o que é equivalente a

$$
f(x(x u)+n(x) u-f(x, 1) x u, w)=0 .
$$

E sendo a forma $f(x, y)$ não-degenerada e $w$ arbitrário segue disto que

$$
x(x u)+n(x) u-f(x, 1) x u=0, \forall x, y, u \in A .
$$


a) $\overline{a+b}=f(1, a+b)-(a+b)=\bar{a}+\bar{b}$.

b) Dado $\lambda \in F, \overline{\lambda a}=f(1, \lambda a)-\lambda a=\lambda \bar{a}$.

c) $\overline{\bar{a}}=f(1, \bar{a})-\bar{a}=f(1, f(1, a)-a)-f(1, a)+a=a$.

d) Sabemos que $\bar{a} \bar{b}=(f(1, a)-a)(f(1, b)-b)$. Mas da equação (1.6) temos que

$$
\begin{aligned}
& (a+b)^{2}+n(a+b)-f(a+b, 1)(a+b)=0 \\
& a^{2}+n(a)-f(a, 1) a=0 \\
& b^{2}+n(b)-f(b, 1) b=0 .
\end{aligned}
$$

Subtraindo da primeira equação obtida as outras duas obtemos

$$
a b+b a-f(1, a) b-f(1, b) a+f(a, b)=0 .
$$

Fazendo $x=y=1, z=a, w=b$ em (1.3) segue que

$$
f(a, b)=f(1, a) f(1, b)-f(1, a b) .
$$

Assim,

$$
a b+b a-f(1, a) b-f(1, b) a+f(1, a) f(1, b)-f(1, a b)=0 .
$$

Isto é,

$$
(f(1, a)-a)(f(1, b)-b)+b a-f(1, a b)=0 .
$$

Logo $\bar{a} \bar{b}=f(1, a b)-b a$. Fazendo $x=y=1, z=b, w=a$ em (1.3) segue que

$$
f(a, b)=f(1, a) f(1, b)-f(1, b a)
$$

Comparando com a equação (1.8) temos que $f(1, a b)=f(1, b a)$. Conseqüentemente $\bar{a} \bar{b}=\overline{b a}$.

Se $\lambda \in F$, então $\bar{\lambda}=f(1, \lambda)-\lambda=\lambda f(1,1)-\lambda=2 \lambda-\lambda=\lambda$. Finalmente, $t(a)=a+\bar{a}=f(1, a) \in F$ e por (1.6) $n(a)=a \bar{a}=f(1, a) a-a^{2} \in F$. Também por (1.6) temos que

$$
a^{2}-t(a) a+n(a)=0
$$


Observemos que $\bar{t} s, t \bar{s} \in T \cap \bar{T} \mathrm{e}$, portanto, $T \bar{T} \subset T \cap \bar{T}$. De onde segue que

$$
\lambda s=(t+\bar{t}) s=t s
$$

e

$$
\mu t=t(s+\bar{s})=t s .
$$

Conseqüentemente $\lambda s=\mu t$ e $s=\left(\lambda^{-1} \mu\right) t$, isto é, o ideal $T$ tem dimensão 1 . Portanto $T$ é isomorfo a $F$ e daí temos que $A=F \oplus F$.

\subsection{O processo de Cayley-Dickson}

Seja $A$ uma álgebra sobre um corpo $F$ com elemento identidade 1 e com uma involução $a \mapsto \bar{a}$, onde para cada $a \in A$ os elementos $a+\bar{a}$ e $a \bar{a}$ pertencem a $F$. Vamos construir uma nova álgebra com involução que contém a álgebra $A$ como uma subálgebra. Em adição, se a dimensão de $A$ é $m$, então a dimensão da nova álgebra será $2 m$.

Seja $\alpha \in F \operatorname{com} \alpha \neq 0$ e seja

$$
(A, \alpha)=\left\{\left(a_{1}, a_{2}\right) \mid a_{i} \in A\right\}
$$

munido das operações de adição componente a componente, multiplicação por escalar e a multiplicação dada por

$$
\left(a_{1}, a_{2}\right)\left(a_{3}, a_{4}\right)=\left(a_{1} a_{3}+\alpha a_{4} \overline{a_{2}}, \overline{a_{1}} a_{4}+a_{3} a_{2}\right) .
$$

Como é fácil ver $(A, \alpha)$ é uma álgebra sobre $F$ e o elemento $(1,0)$ é o elemento identidade para a álgebra $(A, \alpha)$. O conjunto

$$
A^{\prime}=\{(a, 0) \mid a \in A\}
$$


corresponde à forma quadrática $n(x)=x \bar{x}$. Se $x=a_{1}+v a_{2}$ e $y=b_{1}+v b_{2}$, então

$$
f(x, y)=\left(a_{1} \overline{b_{1}}+b_{1} \overline{a_{1}}\right)-\alpha\left(a_{2} \overline{b_{2}}+b_{2} \overline{a_{2}}\right) .
$$

Suponhamos que $f(x, y)=0$ para todo $y \in(A, \alpha)$. Tomando $y=b_{1} \in A$, obtemos

$$
f\left(a_{1}, b_{1}\right)=a_{1} \overline{b_{1}}+b_{1} \overline{a_{1}}=0
$$

para todo $b_{1} \in A$. Como $n(a)$ é estritamente não-degenerada segue que $a_{1}=0$. Da mesma forma, tomando $y=v b_{2}$, como $\alpha \neq 0$ segue que $a_{2}=0$. Portanto $x=0$ e isso significa que a forma $n(x)$ é estritamente não-degenerada em $(A, \alpha)$.

Agora seja $A$ uma álgebra com composição com forma quadrática $n(a)$. Pelo Lema 1.3 , existe uma involução $a \mapsto \bar{a}$ em $A$ tal que $n(a)=a \bar{a}$ e $t(a)=a+\bar{a}$ pertencem ao corpo $F$ para todo elemento $a$ em $A$. Isto significa que podemos aplicar o processo de Cayley-Dickson em $A$. Vamos estão encontrar condições sob as quais a álgebra $(A, \alpha)$ obtida juntamente com a forma $n(x)$ é também uma álgebra com composição.

Lema 1.6. Seja A é uma álgebra com composição. Então $(A, \alpha)$ é uma álgebra com composição se, e somente se, A é associativa.

Prova. Como já foi visto acima a álgebra $(A, \alpha)$ tem um elemento identidade, $(1,0)$. Pelo Lema 1.5 a forma quadrática $n(x)$ é estritamente não-degenerada em $(A, \alpha)$. Então a álgebra $(A, \alpha)$ é uma álgebra com composição se, e somente se, a forma $n(x)$ admite composição. Dado $x=a_{1}+v a_{2}$ temos que

$$
n(x)=n\left(a_{1}+v a_{2}\right)=n\left(a_{1}\right)-\alpha n\left(a_{2}\right) .
$$

Se $y=a_{3}+v a_{4}$, então

$$
\begin{aligned}
n(x y)-n(x) n(y)= & n\left(a_{1} a_{3}+\alpha a_{4} \overline{a_{2}}\right)-\alpha n\left(\overline{a_{1}} a_{4}+a_{3} a_{2}\right) \\
& -\left[n\left(a_{1}\right)-\alpha n\left(a_{2}\right)\right]\left[n\left(a_{3}\right)-\alpha n\left(a_{4}\right)\right] \\
= & \alpha f\left(a_{1} a_{3}, a_{4} \overline{a_{2}}\right)-\alpha f\left(\overline{a_{1}} a_{4}, a_{3} a_{2}\right)
\end{aligned}
$$


e o associador de $X, U, Z$ é dado por

$$
\begin{aligned}
(I I)(U, X, Z)= & (U X) Z-U(X Z) \\
= & (u, x, z)-\alpha[u(s \bar{y})+(\bar{x} s) \bar{t}+(z y) \bar{t}-s(\bar{y} u)-s(\bar{t} \bar{x})-(y \bar{t}) z] \\
& -v[\bar{u}(\bar{x} s)+\bar{u}(z y)+(x z) t-(\bar{x} \bar{u}) s-z(\bar{u} y)-z(x t)+\alpha[(s \bar{y}) t-(t \bar{y}) s]] .
\end{aligned}
$$

Parte 1. Suponhamos que $(A, \alpha)$ é associativa. Como $A$ é uma subálgebra de $(A, \alpha)$, $A$ é associativa. Tomando $t=x=z=0$ e $s=1$ em $(I I)$ segue que

$$
0=(U, X, Z)=\alpha(u \bar{y}-\bar{y} u)
$$

Como $\alpha \neq 0$ temos que $u \bar{y}=\bar{y} u$ para todo $y, u \in A$. Isto é, $A$ é comutativa. Se além disso $(A, \alpha)$ é comutativa, fazendo $u=x=0$ e $t=1$ em $(I)$ temos

$$
0=[U, X]=\alpha(y-\bar{y})
$$

Como $\alpha \neq 0$ temos $y=\bar{y}$ para todo $y \in A$.

Parte 2. Suponhamos que $A$ é associativa e comutativa. Nestas condições

$$
(U, X, Z)=0
$$

Logo $(A, \alpha)$ é associativa. Se além disso $y=\bar{y}$ para todo $y \in A$, então

$$
[U, X]=0
$$

o que nos diz que $(A, \alpha)$ é comutativa.

Das partes 1 e 2 segue que valem (i) e (ii). Para provarmos (iii) basta observar que

$$
\overline{(U, X, Z)}=-(\bar{Z}, \bar{X}, \bar{U})
$$

Assim se provarmos que $(U, X, Z)=0$ teremos que $\overline{(U, X, Z)}=0$. Portanto nosso trabalho se reduz a provar apenas uma das leis alternativas. É claro que $(X, 1, Z)=0$ para todo $X, Z \in(A, \alpha)$. Logo $(X, t(x) 1, Z)=0$ para todo $X, Z \in(A, \alpha)$. Assim

$$
0=(X, X+\bar{X}, Z)=(X, X, Z)+(X, \bar{X}, Z) .
$$




\subsubsection{Tipo II - Álgebra dos números complexos generalizados.}

Seja $F$ um corpo arbitrário e seja $\mu \in F$ tal que $4 \mu+1 \neq 0$. Seja $K(\mu)=F+F v_{1}$, onde $v_{1}^{2}=v_{1}+\mu$. Dado $a=\alpha+\beta v_{1} \in K(\mu), \overline{\alpha+\beta v_{1}}=(\alpha+\beta)-\beta v_{1}$ define uma involução e $n(a)=a \bar{a}$ define uma forma quadrática em $K(\mu)$.

Dados $x, y \in K(\mu)$ temos que

$$
n(x y)=x y \overline{x y}=n(x) n(y) .
$$

Suponhamos que $x=\alpha+\beta v_{1}$. Seja $f(x, y)$ a forma blinear associada a $n(x)$. Suponhamos que $f(x, y)=0$ para todo $y \in K(\mu)$. Em particular se $y=1$ temos

$$
0=f(x, 1)=x+\bar{x}=2 \alpha+\beta \text {. }
$$

Se car $F=2$ então $\beta=0$ e logo $x=\alpha \in F$. Para $y=\alpha-\alpha v_{1}$ temos que

$$
\begin{aligned}
0 & =f\left(\alpha, \alpha-\alpha v_{1}\right)=n\left(\alpha+\alpha-\alpha v_{1}\right)-n(\alpha)-n\left(\alpha-\alpha v_{1}\right) \\
& =n\left(-\alpha v_{1}\right)-n(\alpha)-n\left(\alpha-\alpha v_{1}\right) .
\end{aligned}
$$

Donde segue que

$$
-\alpha^{2}-\alpha^{2} v_{1}=0
$$

Assim $\alpha=0$ e isso nos mostra que $x=0$. Logo, neste caso, $n(x)$ é estritamente não-degenerada. Se car $F \neq 2$ então $x=\alpha-2 \alpha v_{1} \mathrm{e}$

$$
0=f(x, x)=2 n(x)=-2 \alpha^{2}(4 \mu+1) .
$$

Como por hipótese $4 \mu+1 \neq 0$ temos que $\alpha=0$. Logo, também neste caso, $n(x)$ é estritamente não-degenerada. Claramente $1 \in F$ é o elemento identidade de $K(\mu)$. Portanto $K(\mu)$ é uma álgebra com composição.

Observemos que se o polinômio $x^{2}-x-\mu$ é irredutível em $F[x]$ então a álgebra $K(\mu)$ é um corpo (uma extensão quadrática do corpo $F$ ), caso contrário $K(\mu)=F \oplus F$. 
Logo, $n(\delta n-1)=0$ e $\alpha=m^{2}+n^{2} \gamma-m$. Se $\delta=0$ então $n=0$ e $\alpha=m^{2}-m$. Se $\delta \neq 0$ então $n=\delta^{-1}$ e $\alpha=m^{2}+\delta^{-2} \gamma-m$. Em qualquer caso, $4 \alpha+1=1 \neq 0$.

Proposição 1.3. Se a característica do corpo $F$ não é 2, então o elemento $v=v_{1}-\frac{1}{2}$ satisfaz $v^{2}=\alpha$ onde $\alpha=\frac{1}{4}(4 \mu+1) \neq 0$ e neste caso $K(\mu)=(F, \alpha)$. Reciprocamente, se $F$ é um corpo de característica diferente de 2, então a álgebra $(F, \alpha)$ é uma álgebra do tipo II.

Prova. Se car $F \neq 2$, então

$$
v^{2}=v_{1}^{2}-v_{1}+\frac{1}{4}=\frac{1}{4}(4 \mu+1) \neq 0 .
$$

Tomando $\alpha=\frac{1}{4}(4 \mu+1)$ temos que, dados $a_{1}+a_{2} v$ e $a_{3}+a_{4} v \in K(\mu)$,

$$
\left(a_{1}+a_{2} v\right)\left(a_{3}+a_{4} v\right)=a_{1} a_{3}+a_{2} a_{3} v+a_{1} a_{4} v+a_{2} a_{4} v^{2}=\left(a_{1}, a_{2}\right)\left(a_{3} \cdot a_{4}\right) .
$$

Logo $K(\mu)=(F, \alpha)$ para $\alpha=\frac{1}{4}(4 \mu+1)$. Reciprocamente, tomando $v_{1}=v+\frac{1}{2}$ temos que

$$
v_{1}^{2}=\left(\frac{1}{2}, 1\right)\left(\frac{1}{2}, 1\right)=v_{1}+\alpha-\frac{1}{4}
$$

Assim, se fizermos $\mu=\alpha-\frac{1}{4}$, então $4 \mu+1=4 \alpha \neq 0$ e $v_{1}^{2}=v_{1}+\mu$. Além disso

$$
\overline{\alpha+\beta v_{1}}=(\alpha+\beta)-\beta v_{1} .
$$

Portanto $(F, \alpha)$ é uma álgebra do tipo II.

Seja - $: K(\mu) \rightarrow K(\mu)$ uma involução em $K(\mu)$. Como $v_{1}$ é raiz de $x^{2}-x-\mu$, então $\overline{v_{1}}$ também é raiz desse polinômio e daí

$$
x^{2}-x-\mu=\left(x-v_{1}\right)\left(x-\overline{v_{1}}\right)
$$

donde segue que $v_{1}+\overline{v_{1}}=1$. Assim

$$
\overline{v_{1}}=1-v_{1} \text {. }
$$

Isto é, a única involução diferente da identidade em $K(\mu)$ é dada por $\left\{\begin{array}{l}\overline{1}=1 \\ \overline{\tau_{1}}=1-v_{1}\end{array}\right.$. 
$B$ com respeito à forma $f(x, y)$. Isto é,

$$
B^{\perp}=\{a \in A \mid f(a, b)=0, \forall b \in B\} .
$$

Lema 1.7. Seja A uma álgebra com composição e seja $B$ uma subálgebra de A que contém o elemento 1. Então $B^{\perp} B+B B^{\perp} \subseteq B^{\perp}$ e, além disso, para todo a, $b \in B e$ para todo $v \in B^{\perp}$ são válidas as seguintes identidades:

$$
\begin{aligned}
& \left\{\begin{array}{l}
\bar{v}=-v, \\
a v=v \bar{a},
\end{array}\right. \\
& \left\{\begin{array}{l}
a(v b)=v(\bar{a} b), \\
(v b) a=v(a b),
\end{array}\right. \\
& (v a)(v b)=-n(v) b \bar{a} .
\end{aligned}
$$

Prova. Como $v \in B^{\perp}$ temos que

$$
0=f(v, 1)=v \overline{1}+1 \bar{v}=v+\bar{v}
$$

e daí $\bar{v}=-v$. Pelo mesmo motivo

$$
0=f(v, a)=v \bar{a}+a \bar{v}
$$

e portanto $v \bar{a}=-a \bar{v}=-a(-v)=a v$ donde segue (1.10). Pela equação (1.3) temos que

$$
f(a, v b)=f(a 1, v b)=-f(a b, v)+f(a, v) f(1, b)=0
$$

e analogamente que $f(a, b v)=0$. Mas por outro lado,

$$
f(a, v b)=a \overline{v b}+v b \bar{a}
$$

e

$$
f(a, b v)=a \overline{b v}+b v \bar{a} .
$$


Assim,

$$
(v a)(v b)=-v((\bar{a} v) b)-n(v) f(a, 1) b .
$$

Usando novamente (1.10),

$$
(v a)(v b)=-v((v a) b)-n(v) f(a, 1) b
$$

ou ainda,

$$
(v a)(v b)=v(\bar{v}(b a))-n(v) f(a, 1) b .
$$

Mas por (1.13) temos que

$$
v(\bar{v}(b a))=n(v)(b a) .
$$

Donde segue que

$$
(v a)(v b)=-n(v) b \bar{a} .
$$

Completando a prova desse lema.

Lema 1.8. Seja A uma álgebra sobre um corpo $F$ de característica diferente de 2. Seja $f: A \times A \rightarrow F$ uma forma bilinear simétrica. Seja $B$ uma subálgebra de dimensão finita da álgebra $A$ na qual a restrição da forma bilinear $f$ é não-degenerada. Então $B$ possui uma base $\beta=\left\{b_{1}, b_{2}, \ldots, b_{k}\right\}$ tal que

$$
\left\{\begin{array}{l}
f\left(b_{i}, b_{i}\right) \neq 0, \\
f\left(b_{i}, b_{j}\right)=0, \forall i \neq j .
\end{array}\right.
$$

Prova. Vamos provar por indução sobre a dimensão de $B$.

Suponhamos que $\operatorname{dim} B=1$. Se para todo $x \in B$ temos $f(x, x)=0$, então $f(x, y)=0$ para todo $x, y \in B$, e segue que $f$ é degenerada. Logo existe $b_{1} \in B$ tal que $f\left(b_{1}, b_{1}\right) \neq 0$ e como $\operatorname{dim} B=1, \beta=\left\{b_{1}\right\}$ é uma base de $B$ satisfazendo as condições do lema. 
Como $f$ é não-degenerada em $B$ temos $x=0$.

Portanto podemos aplicar a hipótese de indução em $\left\langle b_{1}\right\rangle^{\perp}$. Logo existe uma base $\beta=\left\{b_{2}, b_{3}, \ldots, b_{n}\right\}$ de $\left\langle b_{1}\right\rangle^{\perp}$ tal que

$$
\left\{\begin{array}{l}
f\left(b_{i}, b_{i}\right) \neq 0, \\
f\left(b_{i}, b_{j}\right)=0, \forall i \neq j .
\end{array}\right.
$$

Segue então que $\gamma=\left\{b_{1}, b_{2}, \ldots, b_{n}\right\}$ é uma base de $B$ tal que

$$
\left\{\begin{array}{l}
f\left(b_{i}, b_{i}\right) \neq 0, \\
f\left(b_{i}, b_{j}\right)=0, \forall i \neq j .
\end{array}\right.
$$

Concluindo este lema.

Lema 1.9. Seja A uma álgebra com unidade e com forma quadrática admitindo composição e seja B uma subálgebra de dimensão finita da álgebra A na qual a restrição da forma bilinear $f$ é não-degenerada. Então $A=B \oplus B^{\perp}$.

Prova. Como $B$ tem dimensão finita e $\left.f\right|_{B}$ é não-degenerada, existe uma base $\beta=\left\{b_{1}, b_{2}, \ldots, b_{k}\right\}$ de $B$ tal que

$$
\left\{\begin{array}{l}
f\left(b_{i}, b_{i}\right) \neq 0, \\
f\left(b_{i}, b_{j}\right)=0, \forall i \neq j .
\end{array}\right.
$$

Se $x \in B \cap B^{\perp}$ então

$$
f(x, y)=0, \forall y \in B
$$

Mas isto acontece se, e somente se, $x=0$ pois $\left.f\right|_{B}$ é não-degenerada. Portanto

$$
B \cap B^{\perp}=\{0\}
$$

Dado $a \in A$ podemos escrever

$$
a=\sum_{i=1}^{k} \frac{f\left(a, b_{i}\right)}{f\left(b_{i}, b_{i}\right)} b_{i}+\left(a-\sum_{i=1}^{k} \frac{f\left(a, b_{i}\right)}{f\left(b_{i}, b_{i}\right)} b_{i}\right)
$$


equação acima que $f$ é não-degenerada em $v B$. Seja $B_{1}=B \oplus v B$ e seja $x+v y \in B_{1}$ tal que $f(x+v y, a+v b)=0$ para todo $a+v b \in B_{1}$. Em particular, $f(x+v y, a)=0$ para todo $a \in B$, mas $f(x+v y, a)=f(x, a)+f(v y, a)$ e como $v B \subset B^{\perp}$ temos que $f(v y, a)=0$ para todo $a \in B$. Assim $f(x, a)=0$ para todo $a \in B$ e daí segue que $x=0$. Portanto $x+v y=v y$. Temos também que $f(x+v y, v b)=f(v y, v b)=0$ para todo $v b \in v B \subset B^{\perp}$ e com um raciocínio análogo ao caso anterior concluimos que $v y=0$. Conseqüentemente $\left.f\right|_{B_{1}}$ é não-degenerada. As relações (1.11) e (1.12) mostram que $B_{1}$ é a subálgebra de $A$ obtida de $B$ pelo processo de Cayley-Dickson, pois estas relações mostram que os produtos nessas duas álgebras coincidem. Isto é, $B_{1}=(B, \alpha)$. Como $\bar{v}=-v$ e $\overline{a+v b}=\bar{a}-v b$, a involução induzida em $B_{1}$ pela involução de $A$ coincide com a involução obtida pelo processo de Cayley-Dickson. Observemos que $\operatorname{dim} B_{1}=2 \operatorname{dim} B$. Portanto $B_{1}$ é uma subálgebra de $A$ que satisfaz as mesmas condições de $B$ e então podemos repetir o mesmo processo com a álgebra $B_{1}$.

Vamos agora retornar a álgebra $A$ e considerarmos dois casos separadamente.

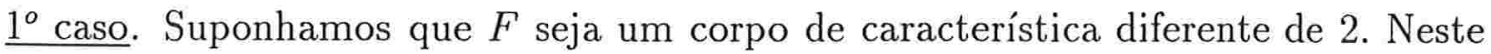
caso a subálgebra $F$ é não-degenerada com respeito a forma $f$ e portanto podemos tomar $B=F$. Se $F \neq A$, então existe uma subálgebra $B_{1}$ do tipo II contida em $A$ e pelo que já vimos $B_{1}$ é associativa e comutativa. Se $B_{1} \neq A$, então existe uma subálgebra $B_{2}$ do tipo III contida em $A$ que é associativa e alternativa. Finalmente, se $B_{2} \neq A$, então $A$ contém uma subálgebra $B_{3}$ do tipo IV que é alternativa e não é associativa. Com isso este processo deve terminar pois, caso contrário, existiria em $A$ uma subálgebra $B_{4}$ que não é uma álgebra com composição, e isso é impossível. Conseqüentemente $A=B_{3}$.

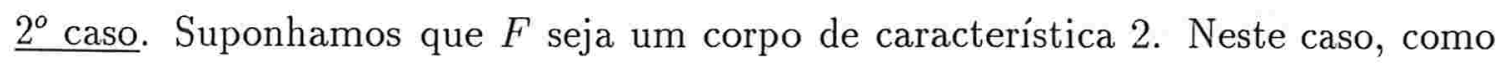
já vimos, $A \neq F$. Suponhamos que $t(x)=f(1, x)=0$ para todo $x \in A \backslash F$. Como $t(\alpha)=2 \alpha=0$ para todo $\alpha \in F$, então $t(x)=f(1, x)=0$ para todo $x \in A$, o que contradiz o fato da forma $f$ ser não-degenerada. Logo existe $a \in A \backslash F$ tal 
Prova. Já vimos que se $n$ admite composição, então $V$ é uma álgebra com composição e então pelo teorema anterior $V$ é de um dos tipos I, II, III, IV. Vamos analisar cada caso.

(i) Se $V$ é do tipo I, então $V$ é um corpo e $\operatorname{dim}_{F} V=1$. Além disso, $n(x)=x \bar{x}=x^{2}$ pois $\bar{x}=x$ para todo $x \in F$.

(ii) Se $V$ é do tipo II, então $V=K(\mu)$. Portanto $\operatorname{dim}_{F} V=2$ e $\left\{1, v_{1}\right\}$ é uma base para $V$ onde $v_{1}^{2}=v_{1}+\mu$ e $4 \mu+1 \neq 0$. Além disso,

$$
n(x)=x \bar{x}=\left(x_{1}+x_{2} v_{1}\right) \overline{\left(x_{1}+x_{2} v_{1}\right)},
$$

e efetuando esse produto temos que

$$
n(x)=x_{1}^{2}+x_{1} x_{2}-\mu x_{2}^{2} .
$$

Se car $F \neq 2$, então consideremos a base $\left\{e_{0}, e_{1}\right\}$ de $V$ onde $e_{0}=1$ e $e_{1}=v_{1}-\frac{1}{2}$. Temos que

$$
e_{1}^{2}=v_{1}^{2}-v_{1}+\frac{1}{4}=\frac{1}{4}(4 \mu+1) \neq 0 .
$$

Chamando $\alpha=\frac{1}{4}(4 \mu+1)$, temos

$$
n(x)=x \bar{x}=\left(x_{1}+x_{2} e_{1}\right) \overline{\left(x_{1}+x_{2} e_{1}\right)},
$$

donde segue que

$$
n(x)=x_{1}^{2}-\alpha x_{2}^{2}
$$

(iii) Se $V$ é do tipo III, então $V=Q(\mu, \beta)=(K(\mu), \beta)$. Portanto $\operatorname{dim}_{F} V=2 \operatorname{dim}_{F} K(\mu)=4$. Se $x \in V$, então $x=a+v_{2} b$ onde $a=x_{1}+x_{2} v_{1} \in K(\mu) \mathrm{e}$ $b=x_{3}+x_{4} v_{1} \in K(\mu)$. Assim $\left\{1, v_{1}, v_{2}, v_{1} v_{2}\right\}$ é uma base de $V$ onde $v_{2}^{2}=\beta \operatorname{com} \beta \neq 0$ (do pocesso de Cayley-Dickson). Como vimos anteriormente $n(x)=n(a)-\beta n(b)$. Portanto

$$
n(x)=x_{1}^{2}+x_{1} x_{2}-\mu x_{2}^{2}-\beta x_{3}^{2}-\beta x_{3} x_{4}+\beta \mu x_{4}^{2} .
$$


que $e_{1}^{2}=\mu \in F$. Vamos construir a tábua de multiplicação de $V$ segundo esta base.

\begin{tabular}{||c|c|c||}
\hline \hline$\bullet$ & 1 & $e_{1}$ \\
\hline 1 & 1 & $e_{1}$ \\
\hline$e_{1}$ & $e_{1}$ & $\mu$ \\
\hline \hline
\end{tabular}

Essa tábua coincide com a tábua de multiplicação da álgebra do tipo II na base para a qual $n(x)=x_{1}^{2}+x_{1} x_{2}-\mu x_{2}^{2}$. Portanto $n(x)$ admite composição em $V$.

(iii) $\operatorname{Se}_{\operatorname{dim}_{F}} V=4$ e $n(x)=x_{1}^{2}+x_{1} x_{2}-\mu x_{2}^{2}-\beta x_{3}^{2}-\beta x_{3} x_{4}+\beta \mu x_{4}^{2}$, então seja $\left\{1, e_{1}, e_{2}, e_{3}\right\}$ com a seguinte tábua de multiplicação:

\begin{tabular}{||c|c|c|c||}
\hline \hline$\bullet$ & $e_{1}$ & $e_{2}$ & $e_{3}$ \\
\hline$e_{1}$ & $\mu$ & $e_{3}$ & $\mu e_{2}$ \\
\hline$e_{2}$ & $-e_{3}$ & $\beta$ & $-\beta e_{1}$ \\
\hline$e_{3}$ & $-\mu e_{2}$ & $\beta e_{1}$ & $-\beta \mu$ \\
\hline \hline
\end{tabular}

Assim a tábua de multiplicação de $V$ nesta base coincide com a tábua de multiplicação da álgebra do tipo II na qual $n(x)=x_{1}^{2}+x_{1} x_{2}-\mu x_{2}^{2}-\beta x_{3}^{2}-\beta x_{3} x_{4}+\beta \mu x_{4}^{2}$. Portanto $n$ admite composição em $V$.

(iv) $\operatorname{Se} \operatorname{dim}_{F} V=8$ e $n(x)=x_{1}^{2}+x_{1} x_{2}-\mu x_{2}^{2}-\beta x_{3}^{2}-\beta x_{3} x_{4}+\beta \mu x_{4}^{2}$, então seja $\left\{1, e_{1}, e_{2}, e_{3}, e_{4}, e_{5}, e_{6}, e_{7}\right\}$ com a seguinte tábua de multiplicação:

\begin{tabular}{||c|c|c|c|c|c|c|c||}
\hline \hline$\bullet$ & $e_{1}$ & $e_{2}$ & $e_{3}$ & $e_{4}$ & $e_{5}$ & $e_{6}$ & $e_{7}$ \\
\hline$e_{1}$ & $\mu$ & $e_{3}$ & $\mu e_{2}$ & $e_{5}$ & $\mu e_{4}$ & $-e_{7}$ & $-\mu e_{6}$ \\
\hline$e_{2}$ & $-e_{3}$ & $\beta$ & $-\beta e_{1}$ & $e_{6}$ & $e_{7}$ & $\beta e_{4}$ & $\beta e_{5}$ \\
\hline$e_{3}$ & $-\mu e_{2}$ & $\beta e_{1}$ & $-\mu \beta$ & $e_{7}$ & $\mu e_{6}$ & $-\beta e_{5}$ & $-\beta \mu e_{4}$ \\
\hline$e_{4}$ & $-e_{5}$ & $-e_{6}$ & $-e_{7}$ & $\gamma$ & $-\gamma e_{1}$ & $-\gamma e_{2}$ & $-\gamma e_{3}$ \\
\hline$e_{5}$ & $-\mu e_{4}$ & $-e_{7}$ & $-\mu e_{6}$ & $\gamma e_{1}$ & $-\mu \gamma$ & $\gamma e_{3}$ & $\mu \gamma e_{2}$ \\
\hline$e_{6}$ & $e_{7}$ & $-\beta e_{4}$ & $\beta e_{4}$ & $\gamma e_{2}$ & $-\gamma e_{3}$ & $-\beta \gamma$ & $-\beta \gamma e_{1}$ \\
\hline$e_{7}$ & $\mu e_{6}$ & $-\beta e_{5}$ & $\mu \beta e_{4}$ & $\gamma e_{3}$ & $-\mu \gamma e_{2}$ & $\beta \gamma e_{1}$ & $\mu \beta \gamma$ \\
\hline \hline
\end{tabular}


e como $n(y) \neq 0$ segue que $n(1)=1$.

Vamos mostrar que $n: A \rightarrow F$ é um monomorfismo de anéis. De fato, dados $x, y \in A$,

$$
n(x y)=n(x) n(y)
$$

e

$$
n(x+y)-n(x)+n(y)=f(x, y)=0
$$

o que nos mostra que $n$ é um homomorfismo de anéis. Suponhamos que $x \in \operatorname{ker} n$. Então $n(x)=0$ e, como $f(x, A)=0$, temos que $x=0$ e podemos concluir que $n$ é injetora.

Logo, $A$ é um anel associativo ( $A$ é isomorfo a um subanel de $F$ ) e também é um anel de integridade. De fato, se $x y=0$ então $n(x) n(y)=n(x y)=0$. Logo $n(x)=0$ ou $n(y)=0$ e, como $n$ é injetora, $x=0$ ou $y=0$. Mais ainda, vamos mostrar em seguida que $A$ é um corpo.

Se $\alpha \in F$ então $n(\alpha)=n(\alpha 1)=\alpha^{2} n(1)=\alpha^{2}$. Se $x \in A$ então $n\left(x^{2}\right)=n(x)^{2} \mathrm{e}$ $n(n(x))=n(x)^{2}$. Como $n$ é injetora temos que $n(x)=x^{2}$. Logo, se $x \in A$ e $x \neq 0$, temos $x\left(n(x)^{-1} x\right)=1$ e então $n(x)^{-1} x$ é o inverso de $x$.

Portanto, $A$ é um corpo. Como $x^{2}=n(x) \in F$ para todo $x \in A$ e car $F=2$, temos que $A$ é uma extensão puramente inseparável de $F$. A dimensão de $A$ sobre $F$ é $2^{t}$ ou infinito. 


\section{Capítulo 2}

\section{Álgebras com Pseudo-Composição}

\subsection{Introdução}

Seja $F$ um corpo de característica diferente de 2 e 3 e seja $A$ uma álgebra comutativa sobre $F$. Dizemos que $A$ é uma álgebra com pseudo-composição se existe uma forma bilinear simétrica $\varphi \neq 0$ tal que

$$
x^{3}=\varphi(x, x) x, \forall x \in A .
$$

A forma bilinear $\varphi$ é obviamente única e a chamaremos de norma de $A$.

O objetivo deste capítulo é estudar álgebras com pseudo-composição sobre um corpo algebricamente fechado de característica diferente de 2 e 3 . Tais álgebras necessariamente têm um idempotente $e$. O objetivo principal é a classificação das álgebras com pseudo-composição que não são do tipo quadrático. Mostraremos que essas álgebras podem ser construídas a partir das álgebras alternativas quadráticas com composição. Especificamente mostraremos:

Teorema 2.1. Seja A uma álgebra com pseudo-composição com idempotente e sobre um corpo algebricamente fechado $F$ de característica diferente de 2 e 3. Então $\operatorname{Rad} \varphi$ é um ideal de $A$, e um dos seguintes casos acontece: 
Assim

$$
2[(y z) x+(x z) y+(x y) z]=2[\varphi(y, z) x+\varphi(x, z) y+\varphi(x, y) z]
$$

isto é,

$$
(y z) x+(x z) y+(x y) z=\varphi(y, z) x+\varphi(x, z) y+\varphi(x, y) z .
$$

Em particular, fazendo $y=x, z=x$ e $x=y$ obtemos

$$
x^{2} y+2 x(x y)=\varphi(x, x) y+2 \varphi(x, y) x .
$$

Definimos o radical de $\varphi$ pelo conjunto

$$
\operatorname{Rad} \varphi=\{x \in A \mid \varphi(x, y)=0, \forall y \in A\}
$$

Proposição 2.1. Qualquer ideal próprio de uma álgebra com pseudo-composição A está contido no radical de sua forma bilinear.

Prova. Seja $B$ um ideal próprio de $A$. Suponhamos que existe $y \in B$ tal que $y \notin \operatorname{Rad} \varphi$.

Para todo $x, z \in A$ temos de (2.2) e (2.3) que

$$
\varphi(x, y) x=\frac{1}{2}\left[x^{2} y+2 x(x y)-\varphi(x, x) y\right] \in B
$$

$\mathrm{e}$

$$
\varphi(y, z) x+\varphi(x, y) z=(y z) x+(x z) y+(x y) z-\varphi(x, z) y \in B .
$$

Se $\varphi(x, y) \neq 0$, então segue da primeira igualdade que $x \in B$. Se $\varphi(x, y)=0$, como $y \notin \operatorname{Rad} \varphi$ existe $z \in A$ tal que $\varphi(y, z) \neq 0$ e neste caso segue da segunda igualdade 
Portanto, podemos escrever (\#) como

$$
\varphi\left(x^{2}, x^{2}\right) x^{2}+2 \varphi\left(x, x^{2}\right) \varphi(x, x) x-\varphi(x, x)^{2} x^{2}-2 \varphi\left(x, x^{2}\right) \varphi(x, x) x=0,
$$

isto é,

$$
\left[\varphi\left(x^{2}, x^{2}\right)-\varphi(x, x)^{2}\right] x^{2}=0
$$

Suponhamos que $x \neq 0$. Se $x^{2} \neq 0$, então $\varphi\left(x^{2}, x^{2}\right)=\varphi(x, x)^{2}$. Se $x^{2}=0$, então

$$
0=x x^{2}=x^{3}=\varphi(x, x) x .
$$

$\operatorname{Logo} \varphi(x, x)=0$ e então $\varphi\left(x^{2}, x^{2}\right)=\varphi(x, x)$ pois $\varphi\left(x^{2}, x^{2}\right)=0$.

Vamos linearizar a equação (2.4). Por um lado, temos

$$
\begin{aligned}
& \varphi\left((2 x+y+z)^{2},(2 x+y+z)^{2}\right)-2 \varphi\left((x+y+z)^{2},(x+y+z)^{2}\right) \\
& -\varphi\left((2 x+y)^{2},(2 x+y)^{2}\right)-\varphi\left((2 x+z)^{2},(2 x+z)^{2}\right)+\varphi\left((y+z)^{2},(y+z)^{2}\right) \\
& -2 \varphi\left((x+z)^{2},(x+z)^{2}\right)+2 \varphi\left((x+y)^{2},(x+y)^{2}\right)+\varphi\left((2 x)^{2},(2 x)^{2}\right)-2 \varphi\left(x^{2}, x^{2}\right) \\
& -\varphi\left(y^{2}, y^{2}\right)-\varphi\left(z^{2}, z^{2}\right)=8\left[\varphi\left(x^{2}, y z\right)+2 \varphi(x y, x z)\right]
\end{aligned}
$$

e, por outro lado,

$$
\begin{aligned}
& \varphi(2 x+y+z, 2 x+y+z)^{2}-2 \varphi(x+y+z, x+y+z)^{2}-\varphi(2 x+y, 2 x+y)^{2} \\
& -\varphi(2 x+z, 2 x+z)^{2}+\varphi(y+z, y+z)^{2}+2 \varphi(x+z, x+z)^{2}+2 \varphi(x+y, x+y)^{2} \\
& +\varphi(2 x, 2 x)^{2}-2 \varphi(x, x)^{2}-\varphi(y, y)^{2}-\varphi(z, z)^{2}= \\
& 8[\varphi(x, x) \varphi(y, z)+2 \varphi(x, y) \varphi(x, z)] .
\end{aligned}
$$

Assim

$$
8\left[\varphi\left(x^{2}, y z\right)+2 \varphi(x y, x z)\right]=8[\varphi(x, x) \varphi(y, z)+2 \varphi(x, y) \varphi(x, z)]
$$

isto é,

$$
\varphi\left(x^{2}, y z\right)+2 \varphi(x y, x z)=\varphi(x, x) \varphi(y, z)+2 \varphi(x, y) \varphi(x, z) .
$$


então

$$
0 \neq \varphi(y, y)^{2}=\varphi\left(y^{2}, y^{2}\right)
$$

por (2.4) e logo $y^{2} \neq 0$ pois $\varphi$ é bilinear. Mas vimos na prova do Lema 2.1 que

$$
y^{2} y^{2}+\varphi(y, y) y^{2}=2 \varphi\left(y, y^{2}\right) y=0
$$

Logo

$$
y^{2} y^{2}=-\varphi(y, y) y^{2} \neq 0
$$

e isto significa que $y^{2} y^{2} \neq 0$ é um múltiplo escalar de $y^{2}$. Seja

$$
e=-\varphi(y, y)^{-1} y^{2} \text {. }
$$

Temos então que

$$
e^{2}=\varphi(y, y)^{-2} y^{2} y^{2}=-\varphi(y, y)^{-1} y^{2}=e
$$

isto é, $e$ é um idempotente.

Se $\varphi\left(x, x^{2}\right)=0$ implica que $\varphi(x, x)=0$, então todo divisor primo de $\varphi\left(x, x^{2}\right)$ também divide $\varphi(x, x)$, mas como $\partial(\varphi(x, x)) \leq 2$ e $\partial\left(\varphi\left(x, x^{2}\right)\right) \leq 3$, temos que as únicas possibilidades são:

$$
\text { (I) }\left\{\begin{array}{c}
\varphi(x, x)=\lambda(x) \mu(x) \\
\varphi\left(x, x^{2}\right)=\alpha \lambda(x) \mu(x)^{2}
\end{array}\right.
$$

onde $\lambda$ e $\mu$ são formas lineares primas entre si e $\alpha \in \bar{F}$, ou

$$
\text { (II) }\left\{\begin{array}{c}
\varphi(x, x)=\lambda(x)^{2} \\
\varphi\left(x, x^{2}\right)=\alpha \lambda(x)^{3}
\end{array}\right.
$$

onde $\lambda$ é uma forma linear e $\alpha \in \bar{F}$. 
isto é,

$$
(\gamma-2 \alpha) \lambda(x) \mu(x)^{2}=-\beta \lambda(x)^{2} \mu(x) .
$$

Se $\gamma-2 \alpha=0$, então

$$
\lambda(x)^{2} \mu(x)=0 .
$$

Logo

$$
\lambda(x)=0 \text { ou } \mu(x)=0
$$

e então

$$
\varphi(x, x)=0, \quad \forall x \in A,
$$

o que é um absurdo, pois $\varphi \neq 0$. Se $\gamma-2 \alpha \neq 0$, então $\mu(x)=k \lambda(x)$. Absurdo! Pois $\lambda$ e $\mu$ são primos entre si.

$$
\text { (ii) } \gamma \lambda(x)^{2} \mu(x)+\beta \lambda(x) \mu(x)^{2}=2 \alpha \lambda(x) \mu(x)^{2} \text {, }
$$

isto é,

$$
(\beta-2 \alpha) \lambda(x) \mu(x)^{2}=-\gamma \lambda(x)^{2} \mu(x),
$$

que como no caso $(i)$, gera um absurdo.

$$
\text { (iii) } \gamma \lambda(x)^{3} \mu(x)+\beta \lambda(x) \mu(x)^{3}=2 \alpha \lambda(x) \mu(x)^{2} \text {, }
$$

isto é,

$$
\mu(x)^{2}(\beta \mu(x)-2 \alpha \lambda(x))=-\gamma \lambda(x)^{3} .
$$

Segue daí que

$$
\begin{gathered}
\mu(x)=r \lambda(x) \text { e } \mu(x)(\beta \mu(x)-2 \alpha \lambda(x))=s \lambda(x)^{2} \quad \text { (absurdo!) } \\
\text { ou } \mu(x)^{2}=r \lambda(x)^{2} \text { e } \beta \mu(x)-2 \alpha \lambda(x)=s \lambda(x) .
\end{gathered}
$$


Seja $e \in \bar{F} \otimes A$ tal que $\lambda(e)=1$. Observemos que $\lambda\left(e^{2}\right)=\lambda(e) \lambda(e)=1$ e, além disso,

$$
e^{2}=e+y \text { para algum } y \in N \text {. }
$$

Mas de (2.1)

$$
e^{3}=\varphi(e, e) e=e
$$

e, por outro lado,

$$
e^{3}=e e^{2}=e(e+y)=e^{2}+e y=e+y+e y .
$$

Logo

$$
e=e+y+e y
$$

e segue que

$$
e y=-y \text {. }
$$

Seja

$$
e_{1}=e+\frac{1}{3} y
$$

Temos

$$
\lambda\left(e_{1}\right)=\lambda\left(e+\frac{1}{3} y\right)=\lambda(e)+\frac{1}{3} \lambda(y)=\lambda(e)=1
$$

e

$$
e_{1}^{2}=e^{2}+\frac{2}{3} e y+\frac{1}{9} y^{2}=e+y-\frac{2}{3} y+\frac{1}{9} y^{2}=e+\frac{1}{3} y+\frac{1}{9} y^{2}=e_{1}+\frac{1}{9} y^{2} .
$$

Usando novamente (2.1) obtemos

$$
e_{1}^{3}=\varphi\left(e_{1}, e_{1}\right) e_{1}=e_{1}
$$


Afirmação 2: $A=F e \oplus S$.

É claro que $F e \oplus S \subset A$. Basta mostrar então que $A \subset F e \oplus S$. Se $a \in A$ então

$$
a=\varphi(e, a) e+a-\varphi(e, a) e .
$$

Como

$$
\begin{aligned}
& \varphi(e, a-\varphi(e, a) e)=\varphi(e, a)-\varphi(e, \varphi(e, a) e)= \\
& \varphi(e, a)-\varphi(e, a) \varphi(e, e)=\varphi(e, a)-\varphi(e, a)=0,
\end{aligned}
$$

temos que $a=\alpha e+s$, onde $\alpha=\varphi(e, a) \in F$ e $s=a-\varphi(e, a) e \in S$. Portanto $A \subset F e \oplus S$, o que prova a afirmação.

Fazendo $x=e$ em (2.6) temos

$$
\varphi(e, e y)=\varphi(e, y)
$$

Isto mostra que $S$ é invariante por $L_{e}$.

Se $a \in A_{\alpha}$, então $e a=\alpha a$. Logo

$$
\varphi(e, a)=\varphi(e, e a)=\varphi(e, \alpha a)=\alpha \varphi(e, a)
$$

isto é,

$$
(1-\alpha) \varphi(e, a)=0
$$

Se $\alpha \neq 1$, então $\varphi(e, a)=0$ e segue que $a \in S$. Portanto

$$
A_{\alpha}=\{z \in S \mid e z=\alpha z\} \quad(\alpha \neq 1)
$$

e conseqüentemente $\varphi\left(e, A_{\alpha}\right)=0$.

Parte 3: Vamos mostrar que $A=F e \oplus A_{\frac{1}{2}} \oplus A_{-1}$. 
Portanto, dado $x \in A$, podemos escrever

$$
x=[a]_{1}+[a]_{\frac{1}{2}}+[a]_{-1}
$$

onde $[a]_{\alpha}$ é a componente de $x$ em relação a $A_{\alpha}$.

Parte 4: Sejam $\alpha, \beta \in\left\{\frac{1}{2},-1\right\}$. Fazendo $x=e, y \in A_{\alpha}$ e $z \in A_{\beta}$ em (2.5) obtemos

$$
\varphi\left(e^{2}, y z\right)+2 \varphi(e y, e z)=\varphi(e, e) \varphi(y, z)+\varphi(e, y) \varphi(e, z)
$$

isto é,

$$
\varphi(e, y z)=(1-2 \alpha \beta) \varphi(y, z)
$$

Mais ainda,

$$
\varphi(y z-\varphi(e, y z) e, e)=\varphi(y z, e)-\varphi(\varphi(e, y z) e, e)=\varphi(y z, e)-\varphi(e, y z) \varphi(e, e)=0
$$

e logo

$$
y z-\varphi(e, y z) \in S
$$

Se $y, z \in A_{\frac{1}{2}}$ então

$$
\begin{gathered}
\varphi\left(y z-\frac{1}{2} \varphi(y, z) e, e\right)=\varphi(y z, e)-\frac{1}{2} \varphi(y, z) \varphi(e, e)= \\
\left(1-2 \frac{1}{2} \frac{1}{2}\right) \varphi(y, z)-\frac{1}{2} \varphi(y, z)=\frac{1}{2} \varphi(y, z)-\frac{1}{2} \varphi(y, z)=0 .
\end{gathered}
$$

Logo

$$
y z-\frac{1}{2} \varphi(y, z) e \in S, \quad \forall y, z \in A_{\frac{1}{2}} .
$$

Se $y \in A_{\frac{1}{2}}$ e $z \in A_{-1}$, então

$$
\begin{gathered}
\varphi(y z-2 \varphi(y, z) e, e)=\varphi(y z, e)-2 \varphi(y, z) \varphi(e, e)= \\
\left(1-2 \frac{1}{2}(-1)\right) \varphi(y, z)-2 \varphi(y, z)=2 \varphi(y, z)-2 \varphi(y, z)=0 .
\end{gathered}
$$


Portanto $v_{\frac{1}{2}}=0, y z=[y z]_{1}+v_{-1}$ e conseqüentemente

$$
A_{\frac{1}{2}}^{2} \subset e F \oplus A_{-1}
$$

Em particular,

$$
y z-\frac{1}{2} \varphi(y, z) e=v_{-1} \in A_{-1}
$$

Se $y \in A_{\frac{1}{2}}$ e $z \in A_{-1}$, então de (2.9) segue que

$$
y z=2 \varphi(y, z) e+v_{\frac{1}{2}}+v_{-1}
$$

onde $v_{\frac{1}{2}} \in A_{\frac{1}{2}}$ e $v_{-1} \in A_{-1}$.

Fazendo $\alpha=\frac{1}{2}$ e $\beta=-1$ em (2.11) temos

$$
\begin{aligned}
0 & =e(y z)-\frac{1}{2} y z-\varphi(y, z) e \\
& =e\left(2 \varphi(y, z) e+v_{\frac{1}{2}}+v_{-1}\right)-\frac{1}{2}\left(2 \varphi(y, z) e+v_{\frac{1}{2}}+v_{-1}\right)-\varphi(y, z) e \\
& =\frac{3}{2} v_{-1} .
\end{aligned}
$$

Portanto $v_{-1}=0$ e logo

$$
y z-2 \varphi(y, z) e=v_{\frac{1}{2}} \in A_{\frac{1}{2}} .
$$

Observemos que

$$
2 z(z y)=2 z\left(2 \varphi(y, z) e+v_{\frac{1}{2}}\right)=4 \varphi(y, z) e z+2 z v_{\frac{1}{2}} .
$$

Mas, como $z \in A_{-1}$,

$$
z v_{\frac{1}{2}}=2 \varphi\left(z, v_{\frac{1}{2}}\right) e+\frac{1}{2} w_{\frac{1}{2}}
$$

onde $w_{\frac{1}{2}} \in A_{\frac{1}{2}}$. Logo

$$
2 z(z y)=-4 \varphi(y, z) z+4 \varphi\left(z, v_{\frac{1}{2}}\right) e+w_{\frac{1}{2}} .
$$


onde $v_{\frac{1}{2}} \in A_{\frac{1}{2}}$ e $v_{-1} \in A_{-1}$. Fazendo $\alpha=\beta=-1$ em (2.11) temos

$$
\begin{aligned}
0 & =e(y z)-2 y z-\varphi(y, z) e \\
& =e\left(-\varphi(y, z) e+v_{\frac{1}{2}}+v_{-1}\right)-2\left(-\varphi(y, z) e+v_{\frac{1}{2}}+v_{-1}\right)-\varphi(y, z) e \\
& =-\varphi(y, z) e+\frac{1}{2} v_{\frac{1}{2}}-v_{-1}+2 \varphi(y, z) e-2 v_{\frac{1}{2}}-2 v_{-1}-\varphi(y, z) e \\
& =\frac{3}{2} v_{\frac{1}{2}}-3 v_{-1}=\frac{1}{2} v_{\frac{1}{2}}-v_{-1}
\end{aligned}
$$

Portanto $v_{\frac{1}{2}}=0, v_{-1}=0$ e logo

$$
y z=-\varphi(y, z) e
$$

Segue então que

$$
A_{-1}^{2} \subset F e .
$$

o que conclui a prova desta proposição.

Suponhamos que $e, f$ são idempotentes não nulos. Digamos que

$$
f=\alpha e+a+b
$$

onde $a \in A_{\frac{1}{2}}$ e $b \in A_{-1}$. Se $e f=0$ então

$$
e f=\alpha e+\frac{1}{2} a-b=0
$$

e $\log \alpha=0, a=0, b=0$. Conseqüentemente $f=0$ o que é um absurdo! Portanto ef $\neq 0$.

Suponhamos $\alpha=0$, isto é, que $f=a+b$, isto é $[f]_{1}=0$. Então

$$
f^{2}=a^{2}+2 a b+b^{2}=a_{-1}+\frac{1}{2} \varphi(a, a) e+2 a b-\varphi(b, b) e
$$

Mas $f^{2}=f=a+b$. Logo

$$
\begin{aligned}
\frac{1}{2} \varphi(a, a) & =\varphi(b, b), \\
a_{-1} & =b \\
2 a b & =a .
\end{aligned}
$$


Prova. Se $x \in A_{\frac{1}{2}}$ então $x^{2} \in A_{-1} \oplus F e$.

Se $\left[x^{2}\right]_{-1}=0$, então $x^{2} \in F e$ e pela Proposição 2.2 (c) temos

$$
x^{2}=\frac{1}{2} \varphi(x, x) e .
$$

Logo

$$
x^{3}=\frac{1}{2} \varphi(x, x) \text { ex }=\frac{1}{4} \varphi(x, x) x=\frac{1}{4} x^{3}
$$

o que é um absurdo, se $x \neq 0$. Portanto

$$
x^{2} \neq 0 \Leftrightarrow\left[x^{2}\right]_{-1} \neq 0
$$

o que conclui a prova deste lema.

\subsection{O caso quadrático}

Seja $A$ uma álgebra sobre um corpo $F$ de dimensão maior que 1 e $e \neq 0$ um idempotente em $A$. Dizemos que $A$ é do tipo e-quadrático se existem uma forma linear $\beta$ e uma forma bilinear simétrica $\gamma$ em $A$ satisfazendo:

$$
x^{2}+\beta(x) x+\gamma(x, x) e=0, \forall x \in A
$$

As formas $\beta$ e $\gamma$ são necessáriamente únicas e as chamamos de $e$-traço e $e$-norma de $x$ em $A$ respectivamente.

Proposição 2.3. São equivalentes:

(i) A é uma álgebra com pseudo-composição satisfazendo $A_{-1}(e)=0$ (respectivamente $A_{\frac{1}{2}}(e)=0$ ).

(ii) A é uma álgebra do tipo e-quadrático. cujo e-traço $\beta$ e a e-norma $\gamma$ satisfazem: $\gamma=0, \beta \neq 0$ (respectivamente $\left.\gamma(e, e)=-3, \beta(x)=-\frac{2}{3} \gamma(e, x)\right)$. 
isto é,

$$
-\varphi(u, u) e=\left(\varphi(x, e)^{2}-\varphi(x, x)\right) e .
$$

Então

$$
\begin{aligned}
& x^{2}+2 \varphi(x, e) x+\left(\varphi(x, x)-4 \varphi(x, e)^{2}\right) e= \\
& \varphi(x, e)^{2} e^{2}+2 \varphi(x, e) e u+u^{2}+2 \varphi(x, e) x+\varphi(x, x) e-4 \varphi(x, e)^{2} e= \\
& \varphi(x, e)^{2} e-2 \varphi(x, e) u+\varphi(x, e)^{2} e-\varphi(x, x) e+2 \varphi(x, e)^{2} e+2 \varphi(x, e) u \\
& +\varphi(x, x) e-4 \varphi(x, e)^{2} e=0 .
\end{aligned}
$$

Logo $A$ é do tipo $e$-quadrático e

$$
\gamma(x, x)=\varphi(x, x)-4 \varphi(x, e)^{2}, \beta(x)=2 \varphi(x, e)
$$

e então,

$$
\varphi(x, x)=\beta(x)^{2}+\frac{1}{2} \beta(e) \gamma(x, x) .
$$

Linearizando $\gamma(x, x)=\varphi(x, x)-4 \varphi(x, e)^{2}$ temos

$$
2 \gamma(x, y)=2 \varphi(x, y)-8 \varphi(x, e) \varphi(y, e) .
$$

Em particular,

$$
\gamma(e, e)=-3 \text { e } \beta(x)=-\frac{2}{3} \gamma(e, x) .
$$

Vamos mostrar agora que $(i i) \Rightarrow(i)$.

$\underline{1 \underline{\underline{o}} \text { caso: }}$ Se $x^{2}=-\beta(x) x$ para todo $x \in A$ e $\beta \neq 0$, então tomando

$$
\varphi(x, x)=\beta(x)^{2}
$$

temos

$$
x^{3}=-\beta(x) x^{2}=\beta(x) \beta(x) x=\beta(x)^{2} x=\varphi(x, x) x .
$$


e logo

$$
x=-\frac{4}{9} \gamma(e, x) e .
$$

Neste caso, $x \in F e \cap A_{\frac{1}{2}}$ e segue então que $x=0$. Portanto $A_{\frac{1}{2}}=0$.

De (2.12) segue que dado $x \in A$

$$
\begin{aligned}
x^{3} & =-\beta(x) x^{2}-\gamma(x, x) x e \\
& =-\beta(x)(-\beta(x) x-\gamma(x, x) e)-\gamma(x, x) x e \\
& =\beta(x)^{2} x+\beta(x) \gamma(x, x) e-\gamma(x, x) x e \\
& =\frac{4}{9} \gamma(e, x)^{2} x-\frac{2}{3} \gamma(e, x) \gamma(x, x) e-\gamma(x, x) e x \\
& =\frac{4}{9} \gamma(e, x)^{2} x-\gamma(x, x)\left(\frac{2}{3} \gamma(e, x) e+e x\right) \\
& =\frac{4}{9} \gamma(e, x)^{2} x-\gamma(x, x)\left(\frac{2}{3} \gamma(e, x) e-\frac{2}{3} \gamma(e, x) e-x\right) \\
& =\left(\frac{4}{9} \gamma(e, x)^{2}+\gamma(x, x)\right) x .
\end{aligned}
$$

Portanto basta tomar

$$
\varphi(x, x)=\frac{4}{9} \gamma(e, x)^{2}+\gamma(x, x)=\beta(x)^{2}+\frac{1}{2} \beta(e) \gamma(x, x)
$$

que obviamente $\varphi$ é bilinear simétrica e $x^{3}=\varphi(x, x) x$.

Suponhamos agora que $A$ é uma álgebra com pseudo-composição do tipo $e$-quadrático com forma bilinear

$$
\varphi(x, x)=\beta(x)^{2}+\frac{1}{2} \beta(e) \gamma(x, x) .
$$

Temos então que

$$
\left(\beta(x)^{2}+\frac{1}{2} \beta(e) \gamma(x, x)\right) x=\varphi(x, x) x=x^{3}=x^{2} x=\left(-\beta(x)^{2} x-\gamma(x, x) e\right) x .
$$

Logo, por (2.14)

$$
\begin{aligned}
\beta(x)^{2} x+\frac{1}{2} \beta(e) \gamma(x, x) x & =-\beta(x)(-\beta(x) x-\gamma(x, x) e)-\gamma(x, x) x e \\
& =\beta(x)^{2} x+\beta(x) \gamma(x, x) e-\gamma(x, x)\left(-\frac{2}{3} \gamma(e, x) e-x\right),
\end{aligned}
$$


Usando a caracterização dada pela Proposição 2.3, vamos mostrar que $\operatorname{Rad} \varphi$ é um ideal das álgebras com pseudo-composição do tipo e-quadrático. Se $x, y \in \operatorname{Rad} \varphi$, então $x+y \in \operatorname{Rad} \varphi$ pois

$$
\varphi(x+y, z)=\varphi(x, z)+\varphi(y, z)=0, \quad \forall z \in A .
$$

Sejam $a \in A$ e $y \in \operatorname{Rad} \varphi$. Quero mostrar que para todo $x \in A$, vale $\varphi(a y, x)=0$. Aplicando $\varphi$ em (2.14) segue que

$$
-2 \varphi(a y, x)=\beta(y) \varphi(a, x)+\beta(a) \varphi(y, x)+2 \gamma(y, a) \varphi(e, x) .
$$

Mas da Proposição 2.3, $\gamma=0$ ou $\gamma(x, y)=\varphi(y, x)-4 \varphi(x, e) \varphi(y, e)=0$. Assim

$$
0=\varphi(y, y)=\beta(y)^{2}+\frac{1}{2} \beta(e) \gamma(y, y)
$$

isto é, $\beta(y)=0$. Logo $\varphi(a y, x)=0$.

Afirmamos também que $\operatorname{Rad} \varphi$ é nilpotente. Mais precisamente, vamos mostrar que $(\operatorname{Rad} \varphi)^{2}=0$. Linearizando $(2.13)$ obtemos

$$
2 \varphi(x, y)=2 \beta(x) \beta(y)+\beta(e) \gamma(x, y) \text {. }
$$

Se $y \in \operatorname{Rad} \varphi$ então

$$
2 \beta(x) \beta(y)+\beta(e) \gamma(x, y)=0 .
$$

Se $\gamma=0$ e $\beta \neq 0$ então $\beta(x) \beta(y)=0$ para todo $x \in A$ e temos que $\beta(y)=0$. Logo, por $(2.12), y^{2}=0$. Se $\gamma(e, e)=-3$ e $\beta(x)=-\frac{2}{3} \gamma(e, x)$ temos

$$
2 \beta(e) \beta(y)+\beta(e) \gamma(e, y)=0
$$

e segue que $\gamma(e, y)=0$ e $\beta(y)=0$. Logo, $\gamma(y, y)=0$ e por (2.12) $y^{2}=0$. Portanto, em qualquer caso, se $y \in \operatorname{Rad} \varphi$ temos que $y^{2}=0$ e isto implica que $(\operatorname{Rad} \varphi)^{2}=0$. 
e logo

$$
v_{\frac{1}{2}}+v_{-1} \in \operatorname{Rad} \varphi
$$

Portanto $x=\alpha e+v$ onde $\alpha \in F$ e $v \in \operatorname{Rad} \varphi$. $\operatorname{Logo} A \subset F e \oplus \operatorname{Rad} \varphi$. Mas, por definição, $F e \oplus \operatorname{Rad} \varphi \subset A$. Conseqüentemente

$$
A=F e \oplus \operatorname{Rad} \varphi
$$

Se $x \in \operatorname{Rad} \varphi$, então $\varphi(x, x)=0$ e daí

$$
x^{3}=\varphi(x, x) x=0 .
$$

Vamos mostrar agora que $\operatorname{Rad} \varphi$ é um ideal de $A$. Seja $y \in \operatorname{Rad} \varphi$, suponhamos que $y=\alpha e+y_{\frac{1}{2}}+y_{-1}$. Temos então que

$$
0=\varphi(y, e)=\varphi\left(\alpha e+y_{\frac{1}{2}}+y_{-1}, e\right)=\alpha \varphi(e, e)+\varphi\left(y_{\frac{1}{2}}, e\right)+\varphi\left(y_{-1}, e\right)=\alpha
$$

e logo

$$
y=y_{\frac{1}{2}}+y_{-1} .
$$

Dado $x=\alpha e+x_{\frac{1}{2}}+x_{-1} \in A$

$$
\begin{aligned}
x y & =\left(\alpha e+x_{\frac{1}{2}}+x_{-1}\right)\left(y_{\frac{1}{2}}+y_{-1}\right) \\
& =\alpha y_{\frac{1}{2}} e+\alpha y_{-1} e+x_{\frac{1}{2}} y_{\frac{1}{2}}+x_{\frac{1}{2}} y_{-1}+x_{-1} y_{\frac{1}{2}}+x_{-1} y_{-1} .
\end{aligned}
$$

Mas da Proposição 2.2 segue que

$$
\begin{aligned}
& x_{\frac{1}{2}} y_{\frac{1}{2}}=v_{-1}+\frac{1}{2} \varphi\left(x_{\frac{1}{2}}, y_{\frac{1}{2}}\right) e=v_{-1}, \\
& x_{\frac{1}{2}} y_{-1}=v_{\frac{1}{2}}, \\
& x_{-1} y_{\frac{1}{2}}=u_{\frac{1}{2}}, \\
& x_{-1} y_{-1}=-\varphi\left(x_{-1}, y_{-1}\right) e=0,
\end{aligned}
$$


Mas

$$
\begin{aligned}
& c\left(\frac{x+c x}{2}\right)=\frac{c x+c(c x)}{2}=\frac{c x+x}{2} \\
& c\left(\frac{x-c x}{2}\right)=\frac{c x-c(c x)}{2}=\frac{c x-x}{2}=-\left(\frac{x-c x}{2}\right) .
\end{aligned}
$$

Portanto $x=u+v$ onde

$$
u=\frac{x+c x}{2} \in U \text { e } v=\frac{x-c x}{2} \in V
$$

e temos que

$$
A_{\frac{1}{2}} \subset U \oplus V
$$

Mas, por definição, $U \oplus V \subset A_{\frac{1}{2}}$. Logo

$$
A_{\frac{1}{2}}=U \oplus V
$$

Se

$$
e^{\prime}=-\frac{1}{2} e+\frac{3}{4} c
$$

e

$$
e^{\prime \prime}=-\frac{1}{2} e-\frac{3}{4} c
$$

então

$$
\begin{aligned}
\left(e^{\prime}\right)^{2} & =\left(-\frac{1}{2} e+\frac{3}{4} c\right)^{2}=\frac{1}{4} e^{2}-\frac{3}{4} c e+\frac{9}{16} c^{2}=\frac{1}{4} e+\frac{3}{4} c-\frac{3}{4} e \\
& =-\frac{1}{2} e+\frac{3}{4} c=e^{\prime} \\
\left(e^{\prime \prime}\right)^{2} & =\left(-\frac{1}{2} e-\frac{3}{4} c\right)^{2}=\frac{1}{4} e^{2}+\frac{3}{4} c e+\frac{9}{16} c^{2}=\frac{1}{4} e-\frac{3}{4} c-\frac{3}{4} e \\
& =-\frac{1}{2} e-\frac{3}{4} c=e^{\prime \prime}
\end{aligned}
$$


(c) Para $u \in U, v \in V$ e $z \in Z$,

$$
\begin{aligned}
u(u v) & =\frac{3}{4} \varphi(u, u) v, \\
v(v u) & =\frac{3}{4} \varphi(v, v) u, \\
u(u z) & =\frac{3}{4} \varphi(u, u) z, \\
v(v z) & =\frac{3}{4} \varphi(v, v) z .
\end{aligned}
$$

Prova. Pela Proposição 2.2 (b) temos que

$$
\varphi\left(A_{\frac{1}{2}}\left(e^{\prime}\right), A_{-1}\left(e^{\prime}\right)\right)=0, \varphi\left(A_{\frac{1}{2}}\left(e^{\prime \prime}\right), A_{-1}\left(e^{\prime \prime}\right)\right)=0 .
$$

Logo pelo que vimos acima $\varphi(U, V)=0, \varphi(V, Z)=0, \varphi(U, Z)=0$.

Se $x \in A_{-1}$, então

$$
x=\frac{3}{4} \varphi(x, c) c-\frac{3}{4} \varphi(x, c) c+x .
$$

Observemos que

$$
\varphi\left(-\frac{3}{4} \varphi(x, c) c+x, c\right)=-\frac{3}{4} \varphi(x, c) \varphi(c, c)+\varphi(x, c)=-\varphi(x, c)+\varphi(x, c)=0 .
$$

Logo

$$
-\frac{3}{4} \varphi(x, c) c+x \in Z
$$

e então

$$
x \in F c \oplus Z .
$$

\section{Conseqüentemente}

$$
A_{-1}=F_{c} \oplus Z .
$$

Também da Proposição 2.2 (a) temos que $A=F e \oplus A_{\frac{1}{2}} \oplus A_{-1}$. Logo

$$
A=F e \oplus F c \oplus U \oplus V \oplus Z
$$


e pela Proprsição 2.2 (d)

$$
z(z u)=\frac{3}{4} \varphi(z, z) u
$$

Linearizando esta equação temos

$$
z(c u)+c(z u)=\frac{3}{2} \varphi(z, c) u=0
$$

e então

$$
-z(c u)=c(z u) .
$$

Como $c u=u$ segue que $c(z u)=-z u$, isto é, $z u \in V$. Logo

$$
U Z \subset V
$$

Da mesma forma, se $v \in V$ e $z \in Z$, então

$$
v z \in A_{-1}\left(e^{\prime}\right) A_{\frac{1}{2}}\left(e^{\prime}\right) \subset A_{\frac{1}{2}}\left(e^{\prime}\right)
$$

e temos que

$$
-z(c v)=c(z v) .
$$

Mas $c v=-v$. Logo $c(z v)=z v$, isto é $z v \in U$. Portanto

$$
V Z \subset U .
$$

Vamos agora provar o ítem (c). Como $u \in U \subset A_{\frac{1}{2}}\left(e^{\prime}\right)$ e $v \in V \subset A_{-1}\left(e^{\prime}\right)$, segue da Proposição 2.2 (d) que

$$
v(v u)=\frac{3}{4} \varphi(v, v) u .
$$

Também $u \in U \subset A_{-1}\left(e^{\prime \prime}\right)$ e $v \in V \subset A_{\frac{1}{2}}\left(e^{\prime \prime}\right)$ implica que

$$
u(u v)=\frac{3}{4} \varphi(u, u) v .
$$


Mas de (2.6) segue que

$$
\varphi\left(u^{2}, z e^{\prime}\right)+\varphi\left(u e^{\prime}, u z\right)=\varphi(u, u) \varphi\left(e^{\prime}, z\right)+2 \varphi\left(u, e^{\prime}\right) \varphi(u, z) .
$$

Logo

$$
\frac{1}{2} \varphi\left(u^{2}, z\right)+\frac{1}{2} \varphi(u, u z)=0
$$

isto é,

$$
\varphi\left(u^{2}, z\right)=-\varphi(u, u z)
$$

Do ítem anterior, $u z \in V \subset A_{-1}\left(e^{\prime}\right)$ e, pela Proposição 2.2 (c),

$$
\varphi(u, u z)=0 .
$$

Portanto

$$
u(u z)=\frac{3}{4} \varphi(u, u) z
$$

Analogamente, fazendo $x=v \in V$ temos $\varphi\left(v^{2}, z\right)=0$. Portanto

$$
v(v z)=\frac{3}{4} \varphi(v, v) z
$$

Completando a prova desta proposição.

Como $U, V, Z$ estão contidos em um $(-1)$-espaço de $e^{\prime \prime}, e^{\prime}, e$ respectivamente, temos pela Proposição 2.2 (d) a seguinte regra de multiplicação:

$$
\left\{\begin{array}{l}
u u^{\prime}=\varphi\left(u, u^{\prime}\right)\left(\frac{1}{2} e+\frac{3}{4} c\right) \\
v v^{\prime}=\varphi\left(v, v^{\prime}\right)\left(\frac{1}{2} e-\frac{3}{4} c\right) \\
z z^{\prime}=-\varphi\left(z, z^{\prime}\right) e
\end{array}\right.
$$

para $u, u^{\prime} \in U, v, v^{\prime} \in V, z, z^{\prime} \in Z$.

Vamos também precisar de algumas propriedades de $\varphi$ derivada da fórmula de pseudo-composição (2.6). 
ou ainda

$$
\varphi(v, v) \varphi(u, u) \varphi\left(\frac{1}{2} e+\frac{3}{4} c, \frac{1}{2} e+\frac{3}{4} c\right)+2 \varphi(u v, u v)=\varphi(v, v) \varphi(u, u) .
$$

Logo

$$
\varphi(u v, u v)=\frac{3}{4} \varphi(v, v) \varphi(u, u)
$$

\subsection{Quando $\varphi$ é zero em $A_{\frac{1}{2}}$ ou em $Z$}

Para nosso principal resultado precisaremos de elementos em $U$ e $Z$ com norma não nula. Se não existir tais elememtos, $\varphi$ é zero em $A_{\frac{1}{2}}$ ou em $Z$. Nestes casos, vamos ver nesta seção que $N:=\operatorname{Rad} \varphi$ é um ideal de $A$ e que $A / N$ é do tipo quadrático.

Proposição 2.5. Seja A uma álgebra com pseudo-composição com forma bilinear $\varphi$. São válidas as seguintes afirmações:

(a) $N=\operatorname{Rad} \varphi$ é sempre uma subálgebra de $A$ e contém as $\left(\frac{1}{2}\right)$-componentes e as $(-1)$-componentes de todos os seus elementos.

(b) Se $\varphi\left(A_{\frac{1}{2}}, A_{\frac{1}{2}}\right)=0$ ou $\varphi(Z, Z)=0$, então $N$ é um ideal de $A$ e $A / N$ é do tipo e-quadrático.

Prova. Vamos provar (a).

Como para todo $n \in N, \varphi(n, e)=0$, temos que $N \subset A_{\frac{1}{2}} \oplus A_{-1}$.

Escrevendo

$$
n=n_{\frac{1}{2}}+n_{-1}
$$

obtemos da Proposição 2.2 (b) que se

$$
x=x_{\frac{1}{2}}+x_{-1}+\alpha e \in A
$$

então

$$
0=\varphi\left(n, x_{\frac{1}{2}}\right)=\varphi\left(n_{\frac{1}{2}}+n_{-1}, x_{\frac{1}{2}}\right)=\varphi\left(n_{\frac{1}{2}}, x_{\frac{1}{2}}\right)+\varphi\left(n_{-1}, x_{\frac{1}{2}}\right),
$$


Vamos agora provar o ítem (b).

Afirmação 1: $A_{\frac{1}{2}}\left(e^{\prime}\right)=U \oplus Z$ e $A_{\frac{1}{2}}\left(e^{\prime \prime}\right)=V \oplus Z$.

De fato, dado $a \in A_{\frac{1}{2}}\left(e^{\prime}\right)$ podemos escrever $a$ na forma

$$
a=\alpha e+\beta c+u+v+z
$$

onde $\alpha, \beta \in F, u \in U, v \in V, z \in Z$, e temos

$$
\begin{aligned}
\frac{1}{2} a & =e^{\prime} a=\alpha e e^{\prime}+\beta c e^{\prime}+u e^{\prime}+v e^{\prime}+z e^{\prime} \\
& =-\frac{1}{2} \alpha e-\frac{3}{4} \alpha c+\frac{1}{2} \beta c-\beta e+\frac{1}{2} u-v+\frac{1}{2} z \\
& =\left(-\frac{1}{2} \alpha-\beta\right) e+\left(-\frac{3}{4} \alpha+\frac{1}{2} \beta\right) c+\frac{1}{2} u-v+\frac{1}{2} z .
\end{aligned}
$$

Segue daí que

$$
\begin{gathered}
-\frac{1}{2} \alpha-\beta=\frac{1}{2} \alpha, \\
-\frac{3}{4} \alpha+\frac{1}{2} \beta=\frac{1}{2} \beta, \\
\frac{1}{2} u=\frac{1}{2} u, \\
-v=\frac{1}{2} v, \\
\frac{1}{2} z=\frac{1}{2} z .
\end{gathered}
$$

Logo, $\alpha=0, \beta=0, v=0$ e então $a=u+z$. Portanto

$$
A_{\frac{1}{2}}\left(e^{\prime}\right) \subset U \oplus Z
$$

Mas $U \oplus Z \subset A_{\frac{1}{2}}\left(e^{\prime}\right)$ por definição. Logo

$$
A_{\frac{1}{2}}\left(e^{\prime}\right)=U \oplus Z
$$

Dado $a \in A_{\frac{1}{2}}\left(e^{\prime \prime}\right)$ podemos escrever $a$ na forma

$$
a=\alpha e+\beta c+u+v+z,
$$


Da Afirmação 1 temos que

$$
\begin{aligned}
\varphi\left(A_{\frac{1}{2}}\left(e^{\prime}\right), A_{\frac{1}{2}}\left(e^{\prime}\right)\right) & =0, \\
\varphi\left(A_{\frac{1}{2}}\left(e^{\prime \prime}\right), A_{\frac{1}{2}}\left(e^{\prime \prime}\right)\right) & =0 .
\end{aligned}
$$

Logo o caso $\varphi(Z, Z)=0$ se reduz ao caso $\varphi\left(A_{\frac{1}{2}}, A_{\frac{1}{2}}\right)=0$ depois de trocado por outro idempotente.

É suficiente mostrar então que $N$ é um ideal quando $\varphi\left(A_{\frac{1}{2}}, A_{\frac{1}{2}}\right)=0$.

Definimos

$$
\begin{aligned}
N_{\lambda} & =\left\{y \in A_{\lambda} \mid \varphi(y, x)=0, \quad \forall x \in A\right\} \\
& =\left\{y \in A_{\lambda} \mid \varphi(y, x)=0, \quad \forall x \in A_{\lambda}\right\} .
\end{aligned}
$$

Afirmação 2: $\operatorname{Rad} \varphi=N_{\frac{1}{2}} \oplus N_{-1}$

De fato, $N_{\frac{1}{2}}, N_{-1} \subset \operatorname{Rad} \varphi$ por definição. Portanto, basta mostrar que $\operatorname{Rad} \varphi \subset N_{\frac{1}{2}} \oplus N_{-1}$. Mas isto é óbvio, pois, dado $x \in \operatorname{Rad} \varphi$,

$$
x=x_{\frac{1}{2}}+x_{-1},
$$

onde $x_{\frac{1}{2}} \in A_{\frac{1}{2}}$ e $x_{-1} \in A_{-1}$, e temos pelo ítem (a) que $x_{\frac{1}{2}}, x_{-1} \in \operatorname{Rad} \varphi$. Logo, $x_{\frac{1}{2}} \in N_{\frac{1}{2}}$, $x_{-1} \in N_{-1}$ e então $x \in N_{\frac{1}{2}} \oplus N_{-1}$, o que completa a prova da afirmação.

$\operatorname{Se~} \varphi\left(A_{\frac{1}{2}}, A_{\frac{1}{2}}\right)=0$ então

$$
A_{\frac{1}{2}}=N_{\frac{1}{2}} \subset N .
$$

Da Proposição 2.2 (c) temos que

$$
\begin{aligned}
A_{-1} N_{\frac{1}{2}} & \subset A_{\frac{1}{2}} \subset N, \\
A_{-1} N_{-1} & \subset \varphi\left(A_{-1}, N_{-1}\right) e=0 .
\end{aligned}
$$

Logo, $A_{-1} N \subset N$. Além disso,

$$
A_{\frac{1}{2}} N_{-1} \subset A_{\frac{1}{2}} \subset N .
$$


Por outro lado,

$$
\begin{aligned}
e a & =e\left(a_{\frac{1}{2}}+a_{-1}+N\right) \\
& =\frac{1}{2} a_{\frac{1}{2}}-a_{-1}+N .
\end{aligned}
$$

Logo

$$
e a-\frac{1}{2} a+a_{-1} \in N
$$

e então $a_{-1} \in N$. Mas $a_{\frac{1}{2}} \in N$, por hipótese, logo $a \in N$. Conseqüentemente $A / N$ não tem $\left(\frac{1}{2}\right)$-espaços.

Afirmação 3: $A / N$ é uma álgebra com pseudo-composição, se considerarmos a aplicação

$$
\begin{gathered}
\bar{\varphi}: A / N \times A / N \rightarrow F \\
(x+N, x+N) \mapsto \varphi(x, x)
\end{gathered}
$$

A aplicação $\bar{\varphi}$ está bem definida pois se $y+N=x+N$ então $x-y \in N$. Daí

$$
0=\varphi(x-y, x+y)=\varphi(x, x)-\varphi(y, y),
$$

isto é,

$$
\varphi(x, x)=\varphi(y, y)
$$

Logo

$$
\bar{\varphi}(x+N, x+N)=\bar{\varphi}(y+N, y+N) .
$$

Dado $x+N \in A / N$

$$
\begin{aligned}
(x+N)^{3} & =x^{3}+N \\
& =\varphi(x, x) x+N \\
& =\varphi(x, x)(x+N) \\
& =\bar{\varphi}(x+N, x+N)(x+N) .
\end{aligned}
$$


Para ver que $L_{a}: V \rightarrow Z$ é um isomorfismo, basta notar que estas funções são inversas uma da outra.

Em particular, $\operatorname{dim} V=\operatorname{dim} Z, Z=a V$ e $V=a Z$.

Da mesma forma, $\varphi(Z, Z) \neq 0$ implica na existência de $z \in Z$ tal que $\varphi(z, z)=\frac{4}{3}$ e $L_{z}: U \rightarrow V$ gera um isomorfismo entre $U$ e $V$.

Para $u, u^{\prime} \in U$ definimos a operação " $\star^{\prime \prime}$ por

$$
u^{\prime} \star u=\left[u^{\prime}(a(z u))\right] z .
$$

Usando a Proposição 2.4 (b) é imediato que $U \star U \subset U$ pois

$$
z u \in V \Rightarrow a(z u) \in Z \Rightarrow u^{\prime}(a(z u)) \in V \Rightarrow\left[u^{\prime}(a(z u))\right] z \in U .
$$

Uma boa propriedade de $(U, \star)$ é dada no lema abaixo.

Lema 2.4. Se $\lambda=\frac{3}{4} \varphi$, então $\lambda\left(u^{\prime} \star u, u^{\prime} \star u\right)=\lambda\left(u^{\prime}, u^{\prime}\right) \lambda(u, u)$.

Prova. De (2.18) e (2.19) segue que

$$
\begin{aligned}
\lambda\left(u^{\prime} \star u, u^{\prime} \star u\right) & =\frac{3}{4} \varphi\left(\left[u^{\prime}(a(z u))\right] z,\left[u^{\prime}(a(z u))\right] z\right) \\
& =\left(\frac{3}{4}\right)^{2} \varphi\left(u^{\prime}(a(z u)), u^{\prime}(a(z u))\right) \varphi(z, z) \\
& =\frac{3}{4} \varphi\left(u^{\prime}(a(z u)), u^{\prime}(a(z u))\right) \\
& =\left(\frac{3}{4}\right)^{2} \varphi(a(z u), a(z u)) \varphi\left(u^{\prime}, u^{\prime}\right) \\
& =\left(\frac{3}{4}\right)^{3} \varphi\left(u^{\prime}, u^{\prime}\right)[\varphi(a, a) \varphi(z u, z u)] \\
& =\left(\frac{3}{4}\right)^{2} \varphi\left(u^{\prime}, u^{\prime}\right) \varphi(z u, z u) \\
& =\left(\frac{3}{4}\right)^{3} \varphi\left(u^{\prime}, u^{\prime}\right)[\varphi(z, z) \varphi(u, u)] \\
& =\left(\frac{3}{4}\right)^{2} \varphi\left(u^{\prime}, u^{\prime}\right) \varphi(u, u) \\
& =\lambda\left(u^{\prime}, u^{\prime}\right) \lambda(u, u) .
\end{aligned}
$$


o que diz que $(U, \star)$ é uma álgebra quadrática.

Afirmação: $u \mapsto \bar{u}=2 \lambda(u, a) a-u$ é uma conjugação em $U$.

De fato, dados $u, u^{\prime} \in U$ e $\alpha \in F$ temos:

$$
\begin{aligned}
\overline{u+u^{\prime}} & =2 \lambda\left(u+u^{\prime}, a\right) a-\left(u+u^{\prime}\right) \\
& =2 \lambda(u, a) a+2 \lambda\left(u^{\prime}, a\right) a-u-u^{\prime} \\
& =2 \lambda(u, a) a-u+2 \lambda\left(u^{\prime}, a\right) a-u^{\prime} \\
& =\bar{u}+\overline{u^{\prime}}, \\
& =2 \lambda(\alpha u, a) a-\alpha u \\
& =2 \alpha \lambda(u, a) a-\alpha u \\
& =\alpha(2 \lambda(u, a) a-u) \\
& =\alpha \bar{u}, \\
\overline{\bar{u}}= & 2 \lambda(u, a) a-u \\
= & 2 \lambda(u, a) \bar{a}-\bar{u} \\
= & 2 \lambda(u, a) a-2 \lambda(u, a) a+u=u .
\end{aligned}
$$

Resta então mostrarmos que esta função é um anti-isomorfismo.

Linearizando $u^{\prime}$ no Lema 2.4 e tomando $a$ como a nova variável temos

$$
2 \lambda\left(a \star u, u^{\prime} \star u\right)=2 \lambda\left(a, u^{\prime}\right) \lambda(u, u)
$$

e logo

$$
\lambda\left(u, u^{\prime} \star u\right)=\lambda\left(a, u^{\prime}\right) \lambda(u, u) .
$$

E fazendo a mesma coisa com $u$ nesta última identidade temos

$$
\lambda\left(u^{\prime} \star u, a\right)+\lambda\left(u^{\prime}, u\right)=2 \lambda\left(a, u^{\prime}\right) \lambda(a, u) .
$$


Usando estas duas últimas identidades e (2.22) temos

$$
\begin{aligned}
u \star\left(u \star u^{\prime}\right) & =u \star\left[u\left(a\left(z u^{\prime}\right)\right)\right] z \\
& =\left[u\left(a\left(z\left[u\left(a\left(z u^{\prime}\right)\right)\right] z\right)\right)\right] z \\
& =\left[u\left(a\left[u\left(a\left(z u^{\prime}\right)\right)\right]\right)\right] z \\
& =-\left[u\left(a\left[a\left(u\left(z u^{\prime}\right)\right)\right]\right)\right] z+\frac{3}{2} \varphi(u, a)\left[u\left(a\left(z u^{\prime}\right)\right)\right] z \\
& =-\left[u\left(u\left(z u^{\prime}\right)\right)\right] z+\frac{3}{2} \varphi(u, a)\left(u \star u^{\prime}\right) \\
& =-\frac{3}{4} \varphi(u, u)\left(z u^{\prime}\right) z+\frac{3}{2} \varphi(u, a)\left(u \star u^{\prime}\right) \\
& =-\frac{3}{4} \varphi(u, u) u^{\prime}+\frac{3}{2} \varphi(u, a)\left(u \star u^{\prime}\right) \\
& =\left[-\frac{3}{4} \varphi(u, u) a+\frac{3}{2} \varphi(u, a) u \star u^{\prime}\right. \\
& =(u \star u) \star u^{\prime} .
\end{aligned}
$$

Para provarmos a lei alternativa à direita, basta lembrar que a conjugação é anti-comutativa e usar a lei alternativa à esquerda. De fato,

$$
\left(u^{\prime} \star u\right) \star u=\overline{\bar{u} \star \overline{\left(u^{\prime} \star u\right)}}=\overline{(\bar{u} \star \bar{u}) \star \overline{u^{\prime}}}=\overline{\overline{u^{\prime}}} \star \overline{(\bar{u} \star \bar{u})}=u^{\prime} \star(u \star u)
$$

Portanto $(U, \star)$ é uma álgebra alternativa quadrática.

Lema 2.6. Dado $u \in U$ qualquer

$$
\varphi(a(z u), z)=\varphi(a, u) .
$$

Prova. Fazendo $y=e$ em (2.5) temos

$$
\varphi\left(x^{2}, e z\right)+2 \varphi(x e, x z)=\varphi(x, x) \varphi(e, z)+2 \varphi(x, e) \varphi(x, z),
$$

isto é,

$$
-\varphi\left(x^{2}, z\right)+2 \varphi(x e, x z)=2 \varphi(x, e) \varphi(x, z) .
$$


Lema 2.7. Se A é uma álgebra alternativa quadrática com unidade sobre um corpo $F$ com traço $t(x)$ e norma $n(x)$ tais que

$$
\begin{aligned}
& t(\alpha)=2 \alpha, \\
& n(\alpha)=\alpha^{2},
\end{aligned}
$$

para todo $\alpha \in F$, então $n$ admite composição.

Prova. Por hipótese, dado $x \in A$

$$
\text { (\#) } \quad x^{2}-t(x) x+n(x)=0 \text {. }
$$

Definimos

$$
\begin{aligned}
f(x, y) & =n(x+y)-n(x)-n(y) \\
& =-x y-y x+t(x) y+t(y) x .
\end{aligned}
$$

Pelo Teorema de Artin (Shafer [Sch66], p. 29) a subálgebra gerada pelos elementos $x, y$ é associativa. Logo

$$
f(x, y) x y=t(x) y x y+t(y) x^{2} y-(x y)^{2}-y x^{2} y
$$

Mas

$$
\begin{aligned}
y x^{2} y & =y(t(x) x-n(x)) y \\
& =t(x) y x y-n(x) y^{2} \\
& =t(x) y x y-n(x)(t(y) y-n(y)) \\
& =t(x) y x y-n(x) t(y) y+n(x) n(y)
\end{aligned}
$$

e

$$
\begin{aligned}
t(y) x^{2} y & =t(y)(t(x) x-n(x)) y \\
& =t(y) t(x) x y-t(y) n(x) y
\end{aligned}
$$


Como $y \notin F$ segue que

$$
n(y)=\frac{\alpha^{2}}{n(x)}
$$

Logo

$$
n(x) n(y)=\alpha^{2}=n(x y) .
$$

Portanto para todo $x, y \in A$

$$
n(x y)=n(x) n(y),
$$

isto é, $n$ admite composição.

Voltando à nossa ágebra $Q$ observemos que

$$
a=a^{2}=2 \lambda(a, a) a-\lambda(a, a) a=\lambda(a, a) a .
$$

Logo

$$
\lambda(a, a)=1 .
$$

Mais ainda, para todo $\alpha \in F$,

$$
\lambda(\alpha, \alpha)=\lambda(\alpha a, \alpha a)=\alpha^{2} \lambda(a, a)=\alpha^{2}
$$

$\mathrm{e}$

$$
2 \lambda(a, \alpha)=2 \alpha \lambda(a, a)=2 \alpha .
$$

Logo $Q$ é uma álgebra que satisfaz as condições do lema anterior. Segue então que

$$
\lambda(x y, x y)=\lambda(x, x) \lambda(y, y)
$$

Seja $P(Q)$ a álgebra consistindo de todas as 5-uplas

$$
\left(\epsilon, \epsilon^{\prime}, x_{1}, x_{2}, x_{3}\right)
$$


Como, por hipótese, $n$ admite composição, para todo $x, y \in A$,

$$
n(x y)=n(x) n(y) .
$$

Linearizando esta equação em $x$ temos

$$
\lambda\left(x y, x^{\prime} y\right)=\lambda\left(x, x^{\prime}\right) n(y)
$$

Fazendo o mesmo em relação a $y$ temos

$$
\lambda\left(x y, x y^{\prime}\right)=\lambda\left(y, y^{\prime}\right) n(x)
$$

Linearizando esta equação em relação a $x$ temos

$$
\lambda\left(x y, x^{\prime} y^{\prime}\right)+\lambda\left(x^{\prime} y, x y^{\prime}\right)=2 \lambda\left(x, x^{\prime}\right) \lambda\left(y, y^{\prime}\right) .
$$

Sabemos que existe $x \in A$ tal que $n(x) \neq 0$. Logo

$$
n(x)=n(a x)=n(a) n(x)
$$

e temos que

$$
n(a)=1 \text {. }
$$

Consideremos a subálgebra $F a$ e observemos que $\operatorname{dim} F a$ é finita sobre $F$. Suponhamos que $\lambda(\alpha a, \beta a)=0$ para todo $\beta a \in F a$. Temos

$$
0=\lambda(\alpha a, \beta a)=\alpha \beta \lambda(a, a)=\alpha \beta n(a)=\alpha \beta
$$

e então $\alpha=0$. Conseqüentemente $\left.\lambda\right|_{F a}$ é não-degenerada. Logo pelo Lema 1.9 temos que

$$
A=F a \oplus F a^{\perp}
$$

Fazendo $x^{\prime}=a$ e $x \in F a^{\perp}$ em (\#) temos

$$
\lambda\left(x y, y^{\prime}\right)+\lambda\left(y, x y^{\prime}\right)=2 \lambda(x, a) \lambda\left(y, y^{\prime}\right)=0 .
$$


Mas

$$
\begin{aligned}
(x \bar{x}) y & =[(\alpha a+z)(\alpha a-z)] y \\
& =\left(\alpha^{2} a-z^{2}\right) y \\
& =\alpha^{2} y-z^{2} y .
\end{aligned}
$$

Portanto

$$
x(\bar{x} y)=(x \bar{x}) y=n(x) y .
$$

Completando a prova deste lema.

Proposição 2.6. A álgebra $P(Q)$ é uma álgebra com pseudo-composição.

Prova. Obviamente $P(Q)$ é comutativa. Se $x=\left(\epsilon, \epsilon^{\prime}, x_{1}, x_{2}, x_{3}\right) \in P(Q)$ então

$$
x^{2}=\left(\eta, \eta^{\prime}, y_{1}, y_{2}, y_{3}\right)
$$

onde

$$
\begin{aligned}
\eta & =\epsilon^{2}-\frac{4}{3}\left(\epsilon^{\prime}\right)^{2}+\frac{2}{3} \lambda\left(x_{1}, x_{1}\right)+\frac{2}{3} \lambda\left(x_{2}, x_{2}\right)-\frac{4}{3} \lambda\left(x_{3}, x_{3}\right), \\
\eta^{\prime} & =-2 \epsilon \epsilon^{\prime}+\lambda\left(x_{1}, x_{1}\right)-\lambda\left(x_{2}, x_{2}\right), \\
y_{1} & =\left(\epsilon+2 \epsilon^{\prime}\right) x_{1}+2 x_{2} \overline{x_{3}}, \\
y_{2} & =\left(\epsilon-2 \epsilon^{\prime}\right) x_{2}+2 x_{1} x_{3}, \\
y_{3} & =-2 \epsilon x_{3}+2 \overline{x_{1}} x_{2} .
\end{aligned}
$$

Definimos

$$
\varphi(x, x)=\epsilon^{2}+\frac{4}{3}\left(\epsilon^{\prime}\right)^{2}+\frac{4}{3} \lambda\left(x_{1}, x_{1}\right)+\frac{4}{3} \lambda\left(x_{2}, x_{2}\right)+\frac{4}{3} \lambda\left(x_{3}, x_{3}\right) .
$$

Claramente $\varphi$ tem as mesmas propriedades de $\lambda$ e, além disso,

$$
x^{3}=x x^{2}=\left(\zeta, \zeta^{\prime}, z_{1}, z_{2}, z_{3}\right)
$$




$$
\begin{aligned}
z_{1}= & \left(\frac{1}{2} \epsilon+\epsilon^{\prime}\right)\left(\left(\epsilon+2 \epsilon^{\prime}\right) x_{1}+2 x_{2} \overline{x_{3}}\right) \\
& +\left(\frac{1}{2} \epsilon^{2}-\frac{2}{3}\left(\epsilon^{\prime}\right)^{2}+\frac{1}{3} \lambda\left(x_{1}, x_{1}\right)+\frac{1}{3} \lambda\left(x_{2}, x_{2}\right)-\frac{2}{3} \lambda\left(x_{3}, x_{3}\right)\right) x_{1} \\
& +\left(-2 \epsilon \epsilon^{\prime}+\lambda\left(x_{1}, x_{1}\right)-\lambda\left(x_{2}, x_{2}\right)\right) x_{1}+x_{2}\left(-2 \epsilon \overline{x_{3}}+2 x_{1} \overline{x_{2}}\right) \\
& +\left(\left(\epsilon-2 \epsilon^{\prime}\right) x_{2}+2 x_{1} x_{3}\right) \overline{x_{3}} \\
= & \frac{1}{2} \epsilon^{2} x_{1}+2 \epsilon \epsilon^{\prime} x_{1}+\epsilon x_{2} \overline{x_{3}}+2\left(\epsilon^{\prime}\right)^{2} x_{1}+2 \epsilon^{\prime} x_{2} \overline{x_{3}}+\frac{1}{2} \epsilon^{2} x_{1}-\frac{2}{3}\left(\epsilon^{\prime}\right)^{2} x_{1} \\
& +\frac{1}{3} \lambda\left(x_{1}, x_{1}\right) x_{1}+\frac{1}{3} \lambda\left(x_{2}, x_{2}\right) x_{1}-\frac{2}{3} \lambda\left(x_{3}, x_{3}\right) x_{1}-2 \epsilon \epsilon^{\prime} x_{1}+\lambda\left(x_{1}, x_{1}\right) x_{1} \\
& -\lambda\left(x_{2}, x_{2}\right) x_{1}-2 \epsilon x_{2} \overline{x_{3}}+2 x_{2}\left(\overline{x_{2}} x_{1}\right)+\epsilon x_{2} \overline{x_{3}}-2 \epsilon^{\prime} x_{2} \overline{x_{3}}+2\left(x_{1} x_{3}\right) \overline{x_{3}} \\
= & \epsilon^{2} x_{1}+\frac{4}{3}\left(\epsilon^{\prime}\right)^{2} x_{1}+\frac{4}{3} \lambda\left(x_{1}, x_{1}\right) x_{1}+\frac{4}{3} \lambda\left(x_{2}, x_{2}\right) x_{1}+\frac{4}{3} \lambda\left(x_{3}, x_{3}\right) x_{1} \\
= & \varphi(x, x) x_{1},
\end{aligned}
$$

$$
\begin{aligned}
z_{2}= & \left(\frac{1}{2} \epsilon-\epsilon^{\prime}\right)\left(\left(\epsilon-2 \epsilon^{\prime}\right) x_{2}+2 x_{1} x_{3}\right)+x_{1}\left(-2 \epsilon x_{3}+2 \overline{x_{1}} x_{2}\right) \\
& +\frac{1}{2}\left(\epsilon^{2}-\frac{4}{3}\left(\epsilon^{\prime}\right)^{2}+\frac{2}{3} \lambda\left(x_{1}, x_{1}\right)+\frac{2}{3} \lambda\left(x_{2}, x_{2}\right)-\frac{4}{3} \lambda\left(x_{3}, x_{3}\right)\right) x_{2} \\
& -\left(-2 \epsilon \epsilon^{\prime}+\lambda\left(x_{1}, x_{1}\right)-\lambda\left(x_{2}, x_{2}\right)\right) x_{2}+\left(\left(\epsilon+2 \epsilon^{\prime}\right) x_{1}+2 x_{2} \overline{x_{3}}\right) x_{3} \\
= & \frac{1}{2} \epsilon^{2} x_{2}-2 \epsilon \epsilon^{\prime} x_{2}+\epsilon x_{1} x_{3}+2\left(\epsilon^{\prime}\right)^{2} x_{2}-2 \epsilon^{\prime} x_{1} x_{3}-2 \epsilon x_{1} x_{3}+2 x_{1}\left(\overline{x_{1}} x_{2}\right) \\
& +\frac{1}{2} \epsilon^{2} x_{2}-\frac{2}{3}\left(\epsilon^{\prime}\right)^{2} x_{2}-\frac{2}{3} \lambda\left(x_{1}, x_{1}\right) x_{2}+\frac{4}{3} \lambda\left(x_{2}, x_{2}\right) x_{2}-\frac{2}{3} \lambda\left(x_{3}, x_{3}\right) x_{2} \\
& +2 \epsilon \epsilon^{\prime} x_{2}+\epsilon x_{1} x_{3}+2 \epsilon^{\prime} x_{1} x_{3}+2\left(x_{2} \overline{x_{3}}\right) x_{3} \\
= & \epsilon^{2} x_{2}+\frac{4}{3}\left(\epsilon^{\prime}\right)^{2} x_{2}+\frac{4}{3} \lambda\left(x_{1}, x_{1}\right) x_{2}+\frac{4}{3} \lambda\left(x_{2}, x_{2}\right) x_{2}+\frac{4}{3} \lambda\left(x_{3}, x_{3}\right) x_{2} \\
= & \varphi(x, x) x_{2},
\end{aligned}
$$


e portanto

$$
A_{-1}=F c \oplus \operatorname{Rad} \varphi_{-1} .
$$

Segue então que $\operatorname{codim}_{A_{-1}} \operatorname{Rad} \varphi_{-1}=1$ o que é um absurdo! $\operatorname{Logo} \varphi(Z, Z) \neq 0$.

Além disso, como $\varphi_{\frac{1}{2}} \neq 0, \varphi(U, U) \neq 0$ ou $\varphi(V, V) \neq 0$. Podemos supor sem perda de generalidade que $\varphi(U, U) \neq 0$.

Sejam $z \in Z$ e $a \in U$ tais que $\varphi(z, z)=\varphi(a, a)=\frac{4}{3}$.

Afirmação 1: $V=z U$ e $Z=a(z U)$

De fato, pela Proposição 2.4, $Z U \subset V$ e para todo $v \in V, v z \in U$. Logo basta mostrar que $V \subset z U$. Dado $v \in V$ segue da Proposição 2.2 (d) que

$$
z(z v)=\frac{3}{4} \varphi(z, z) v=v .
$$

Portanto existe $u \in U$ tal que $v=z u$, o que nos mostra que $V \subset z U$.

Da Proposição 2.4, $a(z U) \subset Z$, logo basta mostrar que $Z \subset a(z U)$.

Seja $z \in Z$ qualquer. Pela Proposição 2.4,

$$
a(z a)=\frac{3}{4} \varphi(a, a) z=z .
$$

Portanto existe $u \in U$ tal que $z=a(z u)$, o que completa a prova desta afirmação.

Podemos então dizer que

$$
A=F e \oplus F c \oplus U \oplus z U \oplus a(z U) .
$$

Seja

$$
\begin{aligned}
\Phi: A & \rightarrow P(Q) \\
\alpha e+\beta c+u_{1}+z u_{2}+a\left(z u_{3}\right) & \mapsto\left(\alpha, \beta, u_{1}, u_{2}, u_{3}\right)
\end{aligned}
$$

Vamos mostrar que $\Phi$ é um isomorfismo. 
Se $y, y^{\prime} \in V$, então de (2.16)

$$
y y^{\prime}=\varphi\left(y, y^{\prime}\right)\left(\frac{1}{2} e-\frac{3}{4} c\right)
$$

e portanto,

$$
\begin{aligned}
\Phi\left(y y^{\prime}\right) & =\Phi\left(\varphi\left(y, y^{\prime}\right)\left(\frac{1}{2} e-\frac{3}{4} c\right)\right)=\varphi\left(y, y^{\prime}\right) \Phi\left(\frac{1}{2} e-\frac{3}{4} c\right) \\
& =\varphi\left(y, y^{\prime}\right)\left(\frac{1}{2},-\frac{3}{4}, 0,0,0\right)=\left(\frac{1}{2} \varphi\left(y, y^{\prime}\right),-\frac{3}{4} \varphi\left(y, y^{\prime}\right), 0,0,0\right) \\
& =\left(\frac{2}{3} \lambda\left(y, y^{\prime}\right),-\lambda\left(y, y^{\prime}\right), 0,0,0\right)=(0,0,0, y, 0)\left(0,0,0, y^{\prime}, 0\right) \\
& =\Phi(y) \Phi\left(y^{\prime}\right) .
\end{aligned}
$$

Se $y, y^{\prime} \in Z$, então de (2.16)

$$
y y^{\prime}=-\varphi\left(y, y^{\prime}\right) e
$$

e portanto,

$$
\begin{aligned}
\Phi\left(y y^{\prime}\right) & =\Phi\left(-\varphi\left(y, y^{\prime}\right) e\right)=-\varphi\left(y, y^{\prime}\right) \Phi(e)=-\varphi\left(y, y^{\prime}\right)(1,0,0,0,0) \\
& =\left(-\varphi\left(y, y^{\prime}\right), 0,0,0,0\right)=\left(-\frac{4}{3} \lambda\left(y, y^{\prime}\right), 0,0,0,0\right)=(0,0,0,0, y)\left(0,0,0,0, y^{\prime}\right) \\
& =\Phi(y) \Phi\left(y^{\prime}\right) .
\end{aligned}
$$

Vamos mostrar agora que a propriedade do homomorfismo vale para produtos mistos.

Dado $z^{\prime}=a\left(z u_{3}\right) \in Z$, segue da proposição $2.4(\mathrm{~d})$ que se $u_{1} \in U$ então

$$
u_{1} z^{\prime}=u_{1}\left[a\left(z u_{3}\right)\right]=z\left\{u_{1}\left[a\left(z u_{3}\right)\right] z\right\}=z\left(u_{1} \star u_{3}\right)
$$

Daí

$$
\begin{aligned}
\Phi\left(u_{1} z^{\prime}\right) & =\Phi\left(z\left(u_{1} \star u_{3}\right)\right)=\left(0,0,0, u_{1} \star u_{3}, 0\right) \\
& =\left(0,0, u_{1}, 0,0\right)\left(0,0,0,0, u_{3}\right)=\Phi\left(u_{1}\right) \Phi\left(z^{\prime}\right) .
\end{aligned}
$$


Logo

$$
u_{3}=-\left(u_{1}\left(a\left(z u_{2}\right)\right)\right) z+2 \lambda\left(a, u_{1}\right) u_{2}
$$

e temos

$$
u_{3}=-u_{1} \star u_{2}+2 \lambda\left(a, u_{1}\right) u_{2}=\overline{u_{1}} \star u_{2} .
$$

Então

$$
\begin{aligned}
\Phi\left(u_{1}\left(z u_{2}\right)\right) & =\Phi\left(a\left(z u_{3}\right)\right)=\left(0,0,0,0, \overline{u_{1}} \star u_{2}\right) \\
& =\left(0,0, u_{1}, 0,0\right)\left(0,0,0, u_{2}, 0\right) \\
& =\Phi\left(u_{1}\right) \Phi\left(z u_{2}\right) .
\end{aligned}
$$

Finalmente vamos analizar produtos da forma $V Z=(z U)[a(z U)]$. Da Proposição $2.2(\mathrm{~d})$, segue que dados $x \in A_{\frac{1}{2}}$ e $z \in A_{-1}$ temos então que

$$
z(z x)=\frac{3}{4} \varphi(z, z) x .
$$

Linearizando esta equação temos

$$
z\left(z_{1} x\right)+z_{1}(z x)=\frac{3}{2} \varphi\left(z, z_{1}\right) x .
$$

Fazendo $z_{1}=a\left(z u_{3}\right), x=u_{2}$ e usando o Lema 2.6

$$
\begin{aligned}
\left(a\left(z u_{3}\right)\right)\left(z u_{2}\right) & =-\left[u_{2}\left(a\left(z u_{3}\right)\right)\right] z+\frac{3}{2} \varphi\left(z, a\left(z u_{3}\right)\right) u_{2} \\
& =-u_{2} \star u_{3}+2 \lambda\left(z, a\left(z u_{3}\right)\right) u_{2} \\
& =-u_{2} \star u_{3}+2 \lambda\left(a, u_{3}\right) u_{2} \\
& =u_{2} \star \overline{u_{3}} .
\end{aligned}
$$

Logo

$$
\begin{aligned}
\Phi\left(\left(a\left(z u_{3}\right)\right)\left(z u_{2}\right)\right) & =\left(0,0, u_{2} \star \overline{u_{3}}, 0,0\right) \\
& =\left(0,0,0, u_{2}, 0\right)\left(0,0,0,0, u_{3}\right) \\
& =\Phi\left(a\left(z u_{3}\right)\right) \Phi\left(z u_{2}\right)
\end{aligned}
$$


isto é,

$$
a_{-1}-\alpha c \in \operatorname{Rad} \varphi_{-1}
$$

Logo, existe $z \in \operatorname{Rad} \varphi_{-1}$ tal que $z=a_{-1}-\alpha c$ e portanto $A_{-1}=F c \oplus \operatorname{Rad} \varphi_{-1}$. Como $A$ é do tipo não-quadrático segue da prova da Proposição 2.4 que $A_{-1}=F c \oplus Z$. Logo $Z=\operatorname{Rad} \varphi_{-1}$. Lembrando que $U V \subset Z$ segue de (2.19) que

$$
0=\varphi(u v, u v)=\frac{4}{3} \varphi(u, u) \varphi(v, v)
$$

e logo

$$
\varphi(u, u)=0 \text { ou } \varphi(v, v)=0
$$

para todo $u \in U, v \in V$. Da demonstração da Proposição 2.5, sabemos que

$$
A_{\frac{1}{2}}\left(e^{\prime}\right)=U \oplus Z \text { e } A_{\frac{1}{2}}\left(e^{\prime \prime}\right)=V \oplus Z
$$

Logo

$$
\varphi\left(A_{\frac{1}{2}}\left(e^{\prime}\right), A_{\frac{1}{2}}\left(e^{\prime}\right)\right)=0 \text { ou } \varphi\left(A_{\frac{1}{2}}\left(e^{\prime \prime}\right), A_{\frac{1}{2}}\left(e^{\prime \prime}\right)\right)=0
$$

e em ambos os casos temos um absurdo! Assim $\operatorname{codim}_{A_{-1}} \operatorname{Rad} \varphi_{-1}>1$.

Portanto $A$ é uma álgebra com pseudo-composição sobre um corpo $F$ com idempotente $e \neq 0$ e tal que $\varphi_{\frac{1}{2}} \neq 0$ e $\operatorname{codim}_{A_{-1}} \operatorname{Rad} \varphi_{-1}>1$. Então pelo Teorema 2.2 $A \cong P(Q)$ onde $Q$ é a álgebra $(U, \star, \lambda)$.

Dos Lemas 2.4 e 2.5 segue que $(U, \star, \lambda)$ é uma álgebra alternativa com unidade, com forma $\lambda=\frac{3}{4} \varphi$ admitindo composição e como por hipótese $\varphi$ é não-degenerada, $(U, \star, \lambda)$ é uma álgebra com composição.

Seja $K$ o radical de $\lambda$ em $Q$. Definimos o conjunto $N \subset P(Q)$ por:

$$
N=\left\{\left(0,0, x_{1}, x_{2}, x_{3}\right) \in P(Q) \mid x_{1}, x_{2}, x_{3} \in K\right\}
$$


Segue que $\lambda\left(x_{1}, y_{1}\right)=0$ e logo $x_{1} \in K$.

Se $y=\left(0,0,0, y_{2}, 0\right) \in P(Q)$, então

$$
0=\varphi(x, y)=\frac{4}{3} \lambda\left(x_{2}, y_{2}\right)
$$

Temos então que $\lambda\left(x_{2}, y_{2}\right)=0$ e logo $x_{2} \in K$.

Se $y=\left(0,0,0,0, y_{3}\right) \in P(Q)$, então

$$
0=\varphi(x, y)=\frac{4}{3} \lambda\left(x_{3}, y_{3}\right) .
$$

Portanto $\lambda\left(x_{3}, y_{3}\right)=0$ e logo $x_{3} \in K$.

Logo $x=\left(0,0, x_{1}, x_{2}, x_{3}\right)$ com $x_{1}, x_{2}, x_{3} \in K$, isto é, $x \in N$. Conseqüentemente $N=\operatorname{Rad} \varphi$.

Vamos mostrar agora que $\operatorname{Rad} \varphi$ é nilpotente quando $Q$ é uma álgebra com composição.

Observemos primeiro que $\operatorname{Rad} \varphi$ é uma nil-álgebra pois se $x \in \operatorname{Rad} \varphi$ então

$$
x^{3}=\varphi(x, x) x=0 .
$$

De (2.2) temos que para todo $x, y \in \operatorname{Rad} \varphi$

$$
\text { (\#) } \quad x^{2} y+2 x(x y)=0 \text {. }
$$

Logo

$$
x\left(x^{2} y\right)+2 x(x(x y))=0 .
$$

Fazendo $y=y x$ em (\#) temos

$$
x^{2}(y x)+2 x(x(y x))=0 .
$$

Segue então que

$$
x^{2}(y x)-x\left(x^{2} y\right)=0 .
$$


Provamos nas Seções 2.3, 2.5 e nesta seção que $\operatorname{Rad} \varphi$ é um ideal de $A$.

Provamos a nilpotência do $\operatorname{Rad} \varphi \operatorname{nos} \operatorname{caos}(\mathrm{a})$ e (c) no final da Seção 2.3 e acima, respectivamente. Quando a dimensão de $\operatorname{Rad} \varphi$ é finita, a nilpotência no caso (b) segue como no caso (c). Fica a pergunta se, no caso (b), $\operatorname{Rad} \varphi$ é nilpotente mesmo quando a dimensão é infinita. 


\section{Referências Bibliográficas}

[MO93] MEYBERG, K. and OSBORN, J. M., Pseudo-composition algebras, Math. Z., 214:67-77, 1993.

[Jac58] JACOBSON, N., Composition algebras and their automorphisms, Rend. Circ. Mat. Palermo, 7(2):55-80, 1958.

[RW88] RÖHRL, H., WALCHER, S., Algebras of complexity one, Algebras, Groups and Geometries, 5:61-107, 1988.

[HK76] HOFFMANN, K, and KUnZE, R., Álgebra Linear, Livros Técnicos e Científicos Editora S. A., primeira edição 1976.

[Jac80] JACOBSON, N., Basic Algebra II, W. H. Freeman an Company, first edition, 1980.

[Sch66] SCHAFER, R. D., An Introduction to Nonassociative Algebras, Academic Press Inc., first edition, 1966.

[ZS82] ZHEVLAKOV, K. A., SLIN 'KO, A. M., SHESTAKOV, I. P. and SHIRSHOV, A. I., Rings That Are Nearly Associative, Academic Press Inc., first edition, 1982. 